\title{
ECONOMIC ANALYSIS FOR THE MOST IMPORTANT PRODUCTS MANUFACTURED FROM EGYPTIAN COTTON: A CASE STUDY: SUOF COMPANY FOR SPINNING AND WEAVING IN ALEXANDRIA
}

Elshahawy, M. I. M.;Dina M. El-Shaaer,and I. M. Ali

Department of Agricultural Economics-Faculty of Agriculture (Saba Basha) - Alexandria University

تحليل إقتصادي لأهم المنتجات المصنعة من القطن المصري (دراسـة حالـة: لشركة

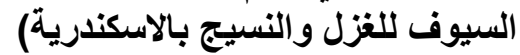

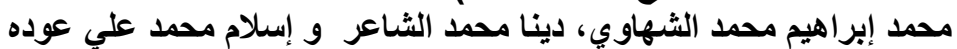

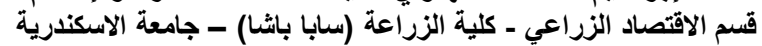

\section{الملخص}

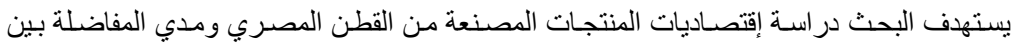

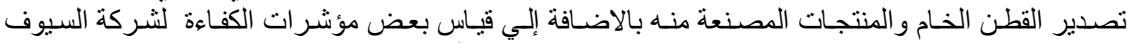

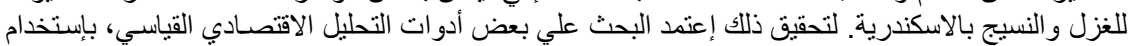
بعض الأساليب الإحصائية والنماذجة الرياضية.

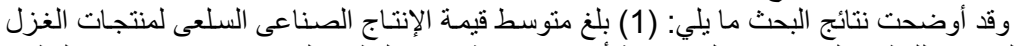

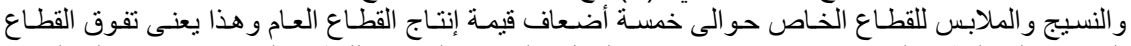

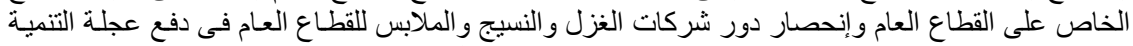

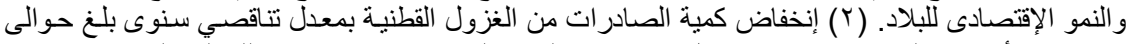

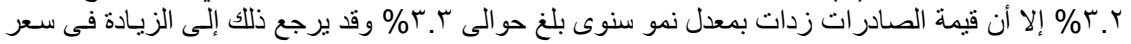

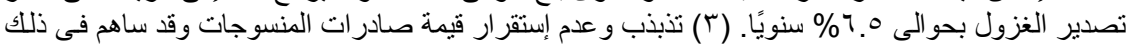

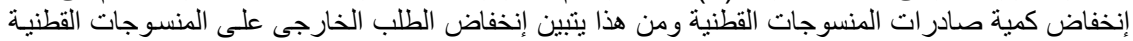

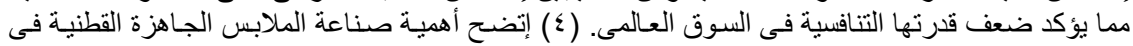

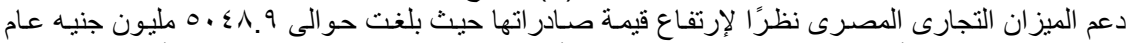

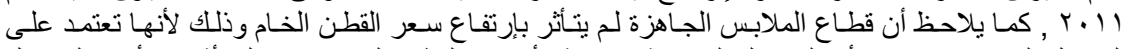

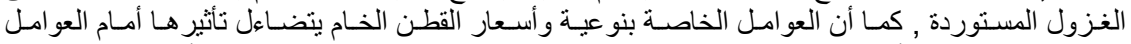

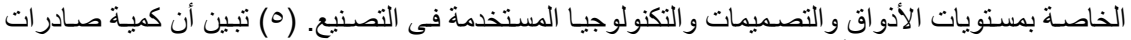

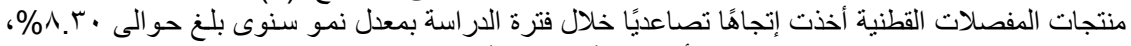

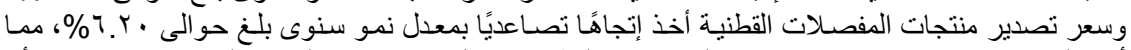

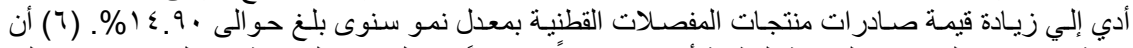

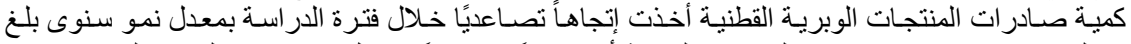

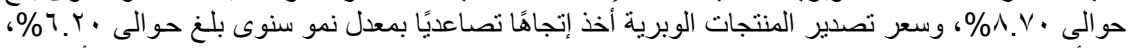

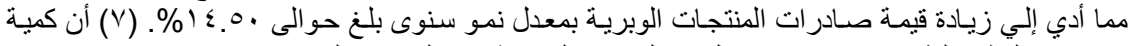

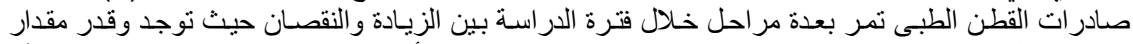

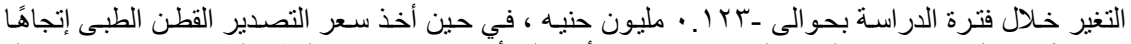

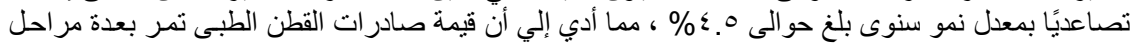

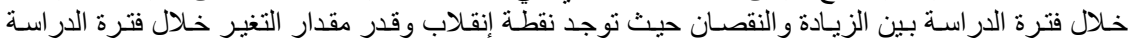

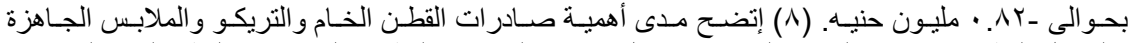

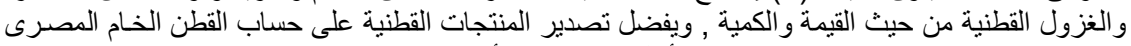

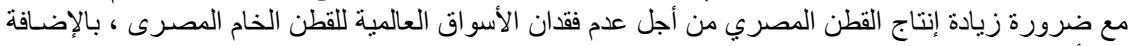

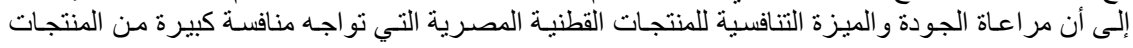


Elshahawy, M. I. M. et al.

القطنية لكل من الصين ودول جنوب شرف آسيا بالإضسافة إلى الدول المتقدمة. (9) بتقدير الكفاءة الإقتصسادية

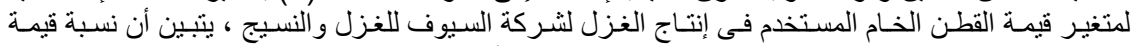

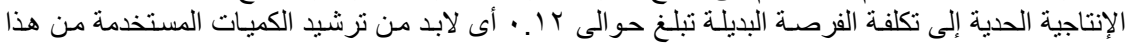

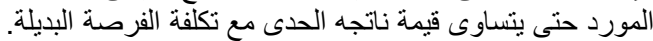

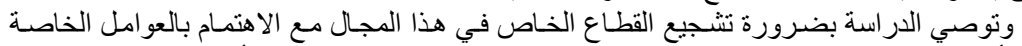

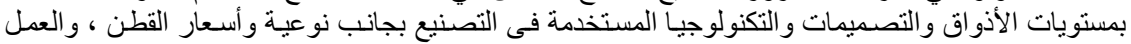

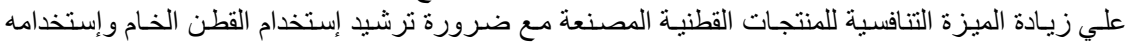

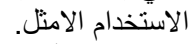

الكلمات الدالة: المنتجات القطنية ، الكفاءة الاقتصادية ، الأثر المشترك ، التشريعات والاتفاقيات الدولية

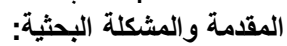

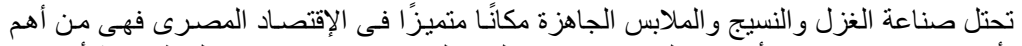

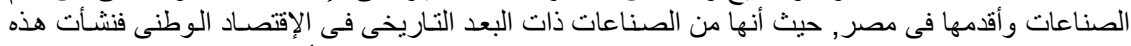

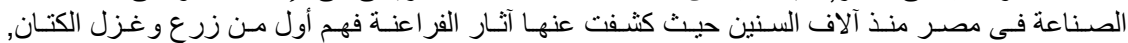

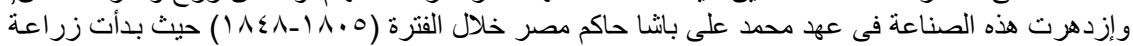

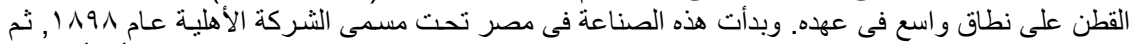

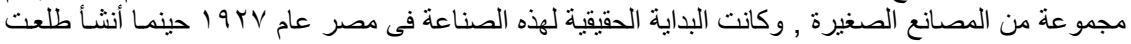

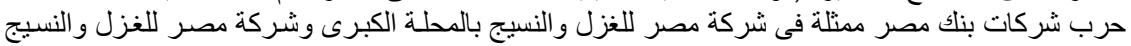

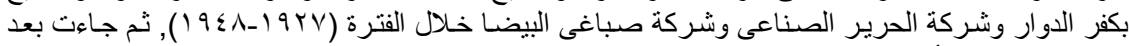

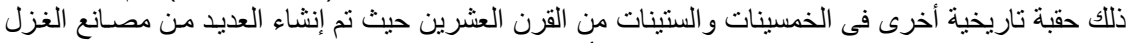

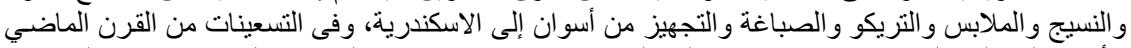

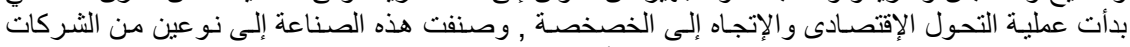

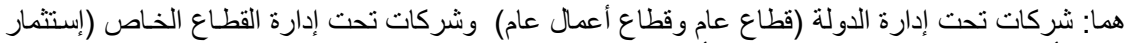
رجال أعمال و إستثمار مشترك وارة وإستثمار أجنبى).

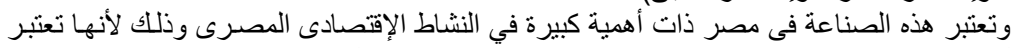

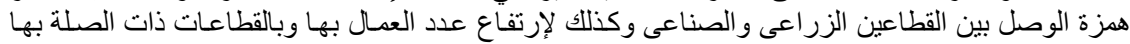

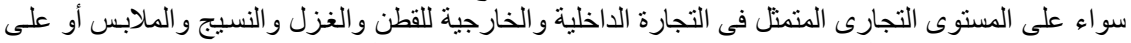

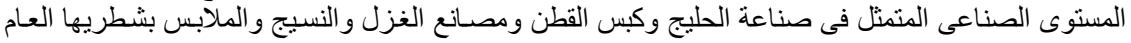

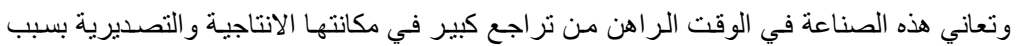

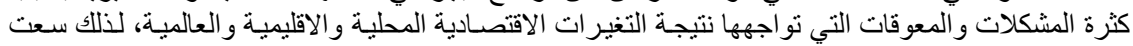

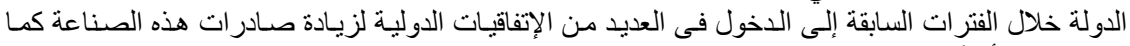

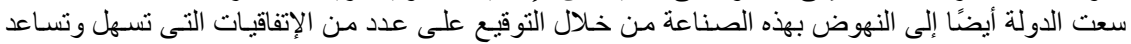

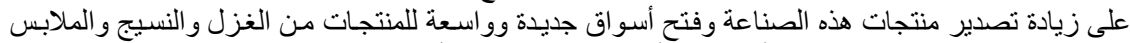

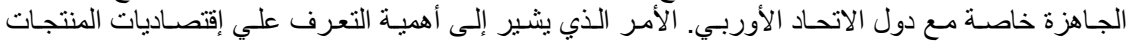

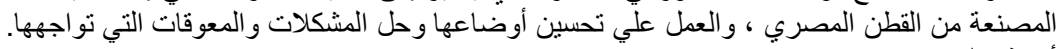
أهداف البحث

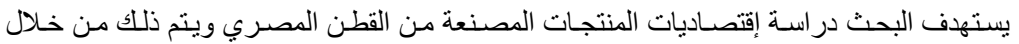

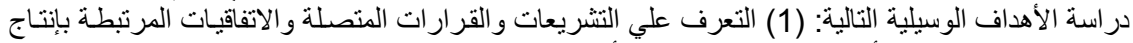

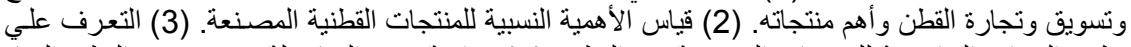

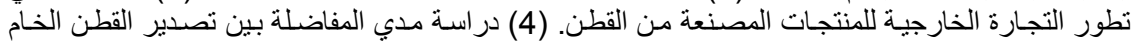

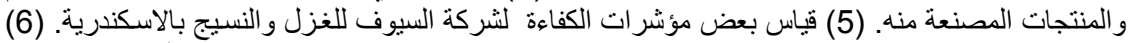

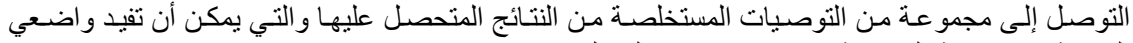
السياسة الاقتصادية الزر اعية في مصر في هذا المجال. 


\section{الأسلوب البحثي}

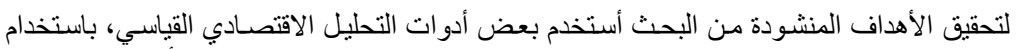

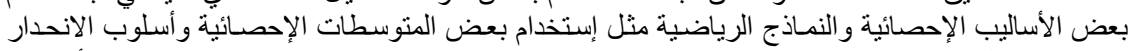

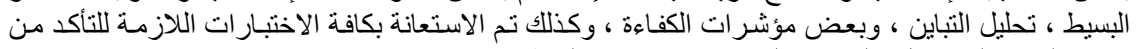

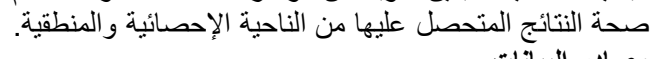

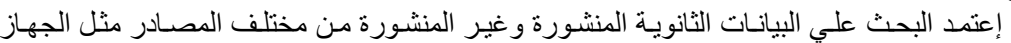
مصادر البيانات

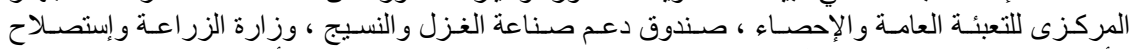

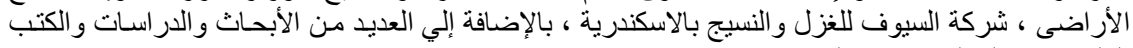
العلمية ذات الصلة بموضع البحث.

\section{النتائج البحثية والمناقشة}

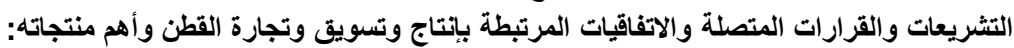

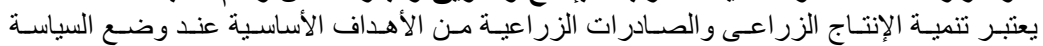

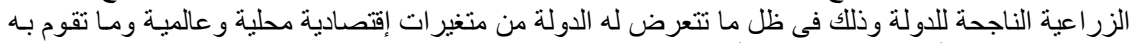

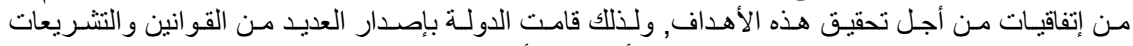

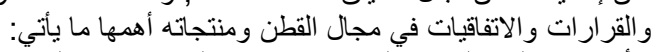

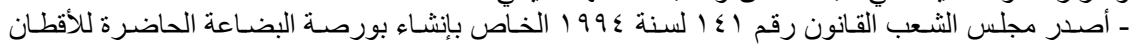

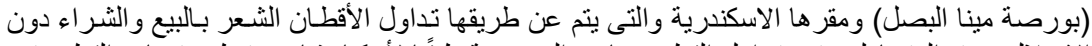

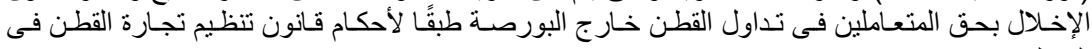

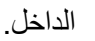

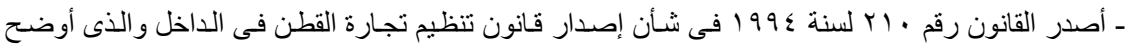

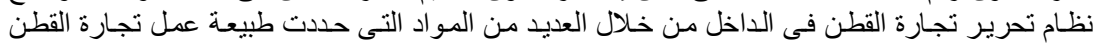

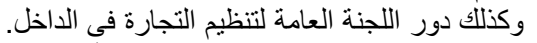

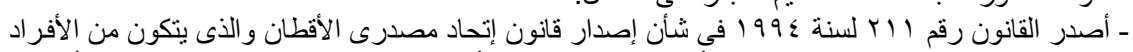

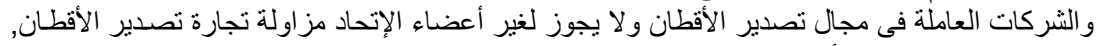

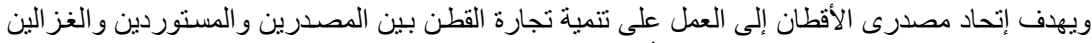

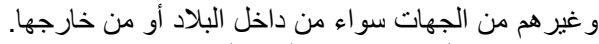

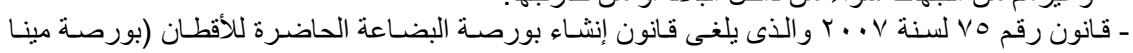

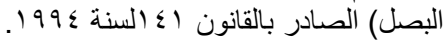

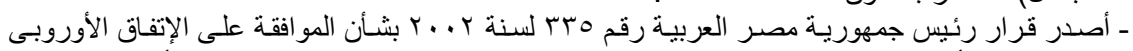

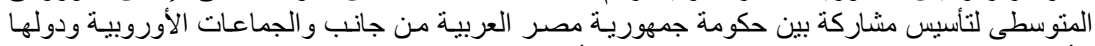

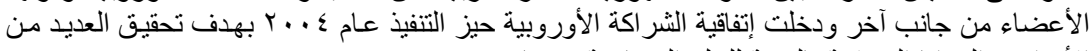

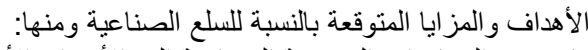

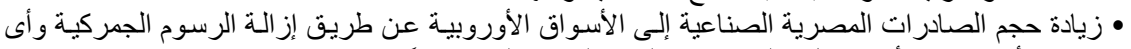

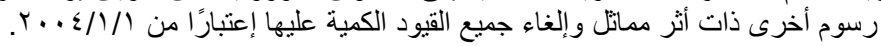

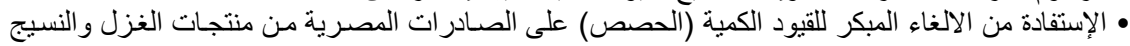

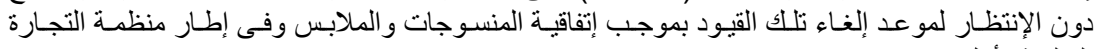

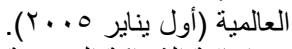

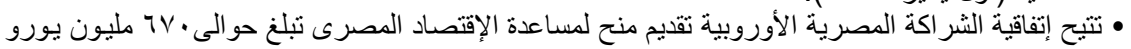

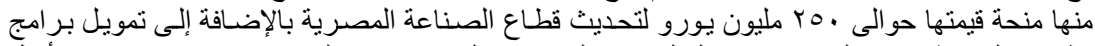

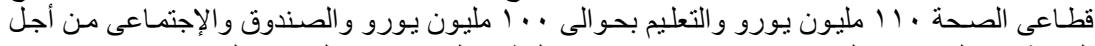

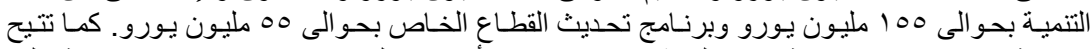

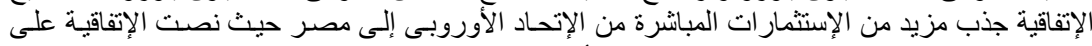
إتخاذ الإجر اءات اللازمة لتشجيع إنتقال رؤوس الأمو ال بين الجانبين وذلك بهدف تشجيع الإستثمار. 


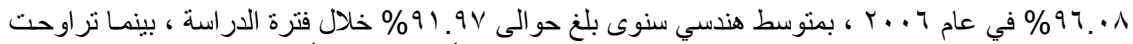

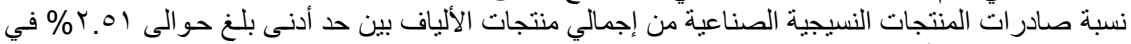

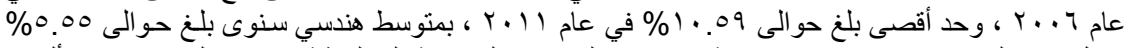

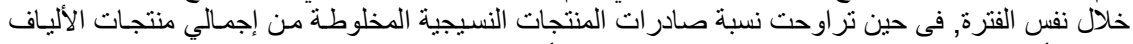

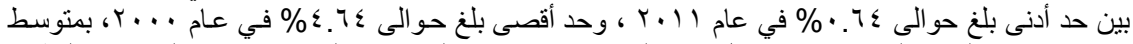

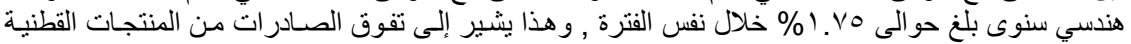
النسيجية على باقى الصنادر اتى النسيجية الأخرى.

جدول رقم (ץ): الأهمية النسبية لصـادرات مصر من المنتجات القطنيـة بالمقارنـة بمنتجات الألياف الأخرى

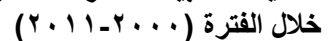

\begin{tabular}{|c|c|c|c|c|c|c|c|c|}
\hline \multicolumn{2}{|c|}{ الإجمالى } & \multicolumn{2}{|c|}{ صادرات المنتجات المخلوطة } & \multicolumn{2}{|c|}{ صادرات المنتجات الصناعية } & \multicolumn{2}{|c|}{ صادنرات الصنتجةت } & \multirow{3}{*}{ 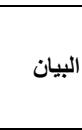 } \\
\hline الأهمية & أقيمة & الأهمية & ألقيمة & الأهمية & القيمة & الأهمية & القيمة & \\
\hline النسبية & (ألف جنيه) & النسبية & (ألف جنيه) & النسبية & (ألف جنيه) & النسبية & (ألف جنيه) & \\
\hline 100 & 3185751 & 4.64 & 147667 & 7.11 & 226633 & 88.25 & 2811451 & 2000 \\
\hline 100 & 3214560 & 3.24 & 104180 & 6.14 & 197351 & 90.62 & 2913065 & 2001 \\
\hline 100 & 3579415 & 3.05 & 109279 & 6.78 & 242610 & 90.17 & 3227526 & 2002 \\
\hline 100 & 5223901 & 2.22 & 116219 & 5.55 & 289945 & 92.22 & 4817737 & 2003 \\
\hline 100 & 5680989 & 2.16 & 122692 & 3.91 & 222270 & 93.93 & 5336027 & 2004 \\
\hline 100 & 5505311 & 2.18 & 120148 & 3.45 & 189899 & 94.37 & 5195264 & 2005 \\
\hline 100 & 6920163 & 1.40 & 97202 & 2.51 & 174002 & 96.08 & 6648959 & 2006 \\
\hline 100 & 7350527 & 1.59 & 117079 & 4.41 & 324275 & 94.00 & 6919173 & 2007 \\
\hline 100 & 9186711 & 1.37 & 125992 & 5.01 & 460543 & 93.62 & 8600176 & 2008 \\
\hline 100 & 9515331 & 0.77 & 73635 & 7.55 & 718749 & 91.67 & 8722947 & 2009 \\
\hline 100 & 11817168 & 1.15 & 135936 & 8.60 & 1016777 & 90.25 & 10664455 & 2010 \\
\hline 100 & 13887320 & 0.64 & 89400 & 10.59 & 1470291 & 88.77 & 12327629 & 2011 \\
\hline 100 & 7088929 & 1.75 & 113285.75 & 5.55 & 461112.08 & 91.97 & 6515367 & الكتوسط \\
\hline
\end{tabular}

المصدر: جمعت وحسبت من بيانات صندوق دعم صناعة الغزل والنسيج , الإدارة العامة للبحوث, النشرة الربع سنوية , أعداد متفرقة.

تطور التجارة الخارجية للمنتجات المصنعة من القطن:

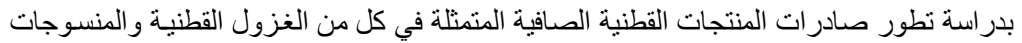
القطنية و التزيكو والملابس الجاهزة و المفصلات و المنتجات الوبرية و القطن الطبى، إنضح الفئح ما يلي: ـ تطور صادرات الغزول القطنية:

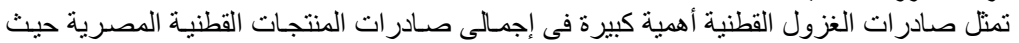

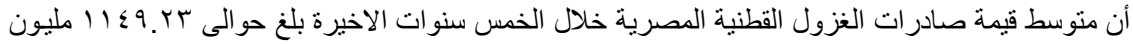

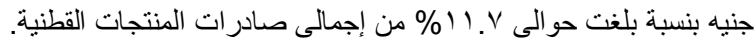

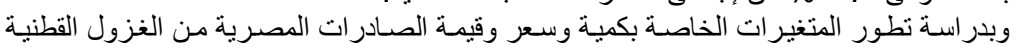

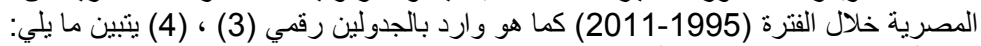

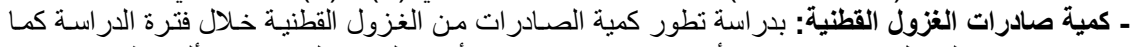

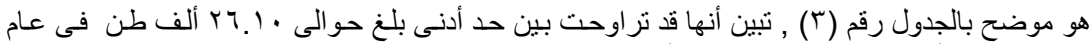

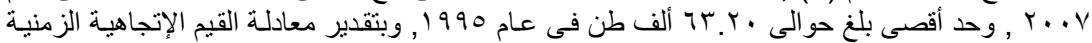

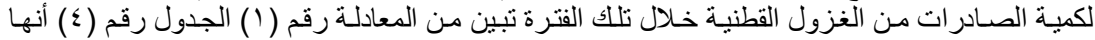

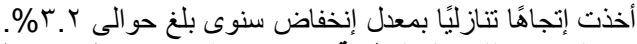

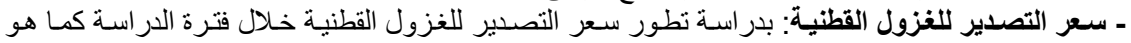

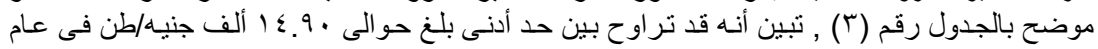

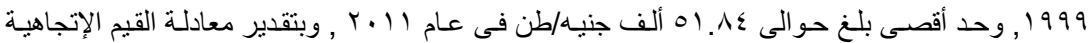

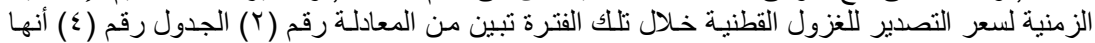

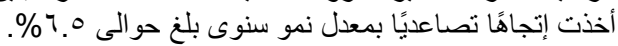




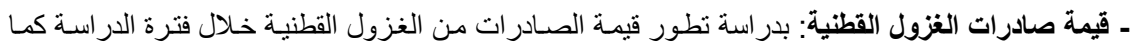

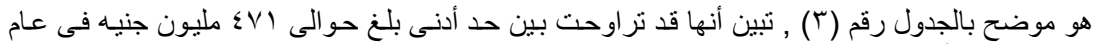

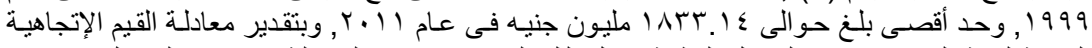

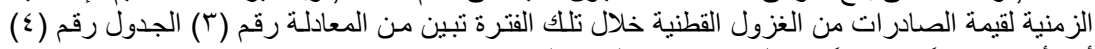

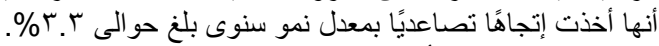

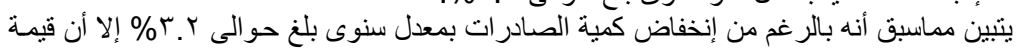

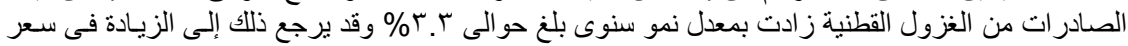

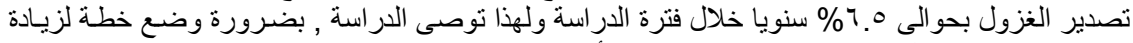

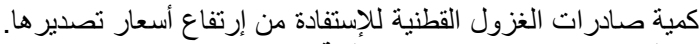
ـ تطور صادرات المنسوجات القطنية:

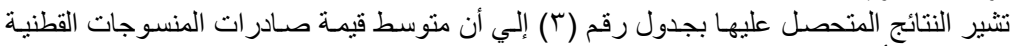

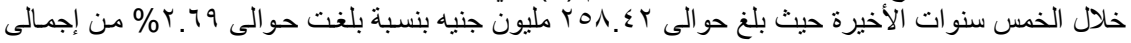
قيمة صادر ات المنتجات القطنية المصنرية

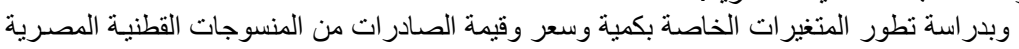

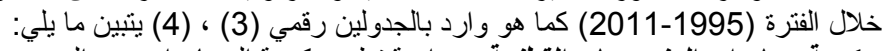

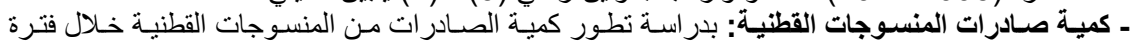

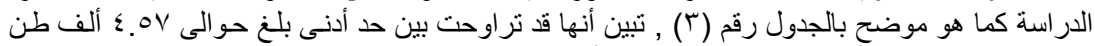

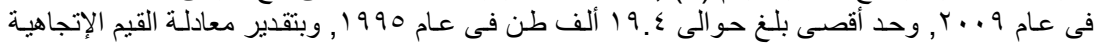

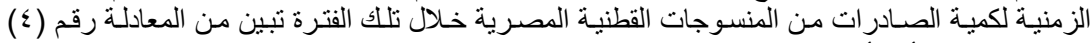

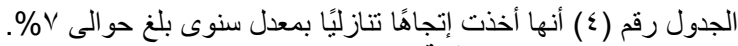

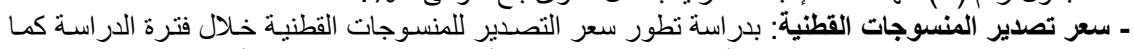

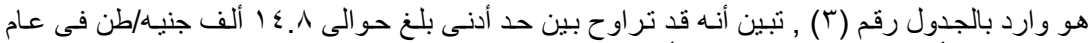

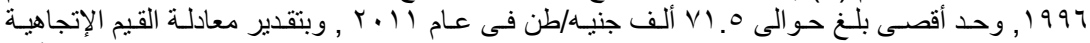

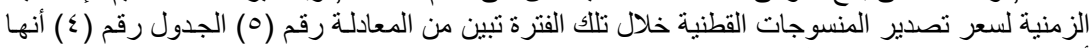

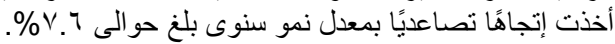

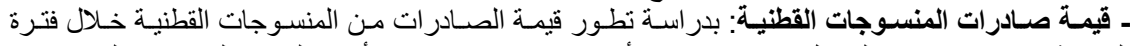

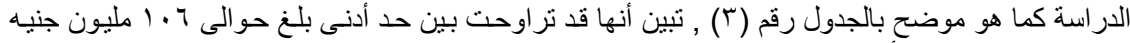

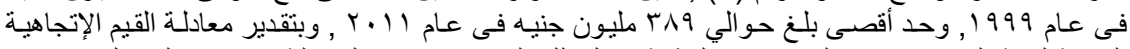

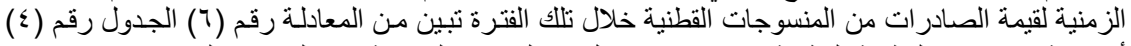

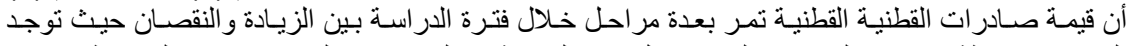

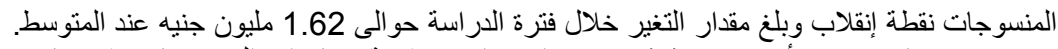

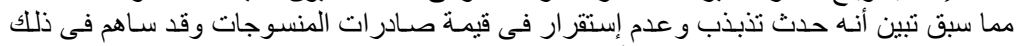

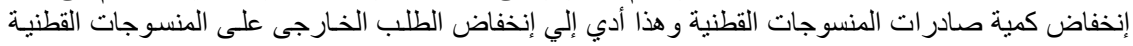
مما يؤكد ضعف قدرثها التنافسية فى السوق العالمى. 


\begin{tabular}{|c|c|c|c|c|c|c|c|c|}
\hline \multicolumn{4}{|c|}{ المنسوجات القطنية } & \multicolumn{4}{|c|}{ الغزول القطنية } & \multirow[b]{2}{*}{ السنة } \\
\hline قيمة صالادمالى & (مليون) & (ألف جنيه) & (ألف طنة) & \% لإجمالى قيمة & 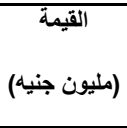 & (ألف جنيه) & (ألف طنة) & \\
\hline 11.96 & r. T.Or & 15.80 & 19.40 & 39.21 & $1 \cdots \varepsilon . \wedge \Lambda$ & 15.90 & 63.20 & 1995 \\
\hline 11.05 & ros.0T & 14.80 & 17.20 & 28.43 & 700.1 & 15.90 & 41.20 & 1996 \\
\hline 11.08 & $r .1 . \varepsilon \varepsilon$ & 15.70 & 19.20 & 34.53 & १५१... & 15.00 & 62.60 & 1997 \\
\hline 5.94 & 10r.I & 17.60 & 8.70 & 29.61 & ع.1. & 15.80 & 48.30 & 1998 \\
\hline 4.66 & $1 \cdot 7.1$ & 15.60 & 6.80 & 20.68 & $\varepsilon V \cdot \lambda \varepsilon$ & 14.90 & 31.60 & 1999 \\
\hline 6.85 & 194.07 & 16.60 & 11.60 & 20.52 & OVT.Ar & 15.10 & 38.20 & 2000 \\
\hline 4.81 & $1 \leq \cdot .14$ & 17.30 & 8.10 & 20.05 & O^E.1. & 16.50 & 35.40 & 2001 \\
\hline 3.59 & $110 . \wedge \varepsilon$ & 18.10 & 6.40 & 19.68 & O Tro & 17.50 & 36.30 & 2002 \\
\hline 3.45 & 111.01 & 17.70 & 6.30 & 19.68 & ס א. & 17.50 & 36.30 & 2003 \\
\hline 2.73 & $1 \leqslant 0.10$ & 26.50 & 5.50 & 16.40 & Nvo.1 & 26.60 & 32.90 & 2004 \\
\hline 2.91 & $101 . T$. & 25.20 & 6.00 & 14.71 & $V T \leqslant . \leqslant T$ & 24.90 & 30.70 & 2005 \\
\hline 2.33 & $10 \leq . \wedge$. & 25.80 & 6.00 & 12.42 & AY7.07 & 28.80 & 28.70 & 2006 \\
\hline 1.87 & $|r q .4|$ & 19.30 & 6.70 & 10.01 & 791.70 & 26.50 & 26.10 & 2007 \\
\hline 2.56 & $r r \cdot . \leqslant V$ & 31.86 & 6.92 & 8.97 & $V \times 1 . \leqslant 9$ & 28.68 & 26.90 & 2008 \\
\hline 1.93 & IYA.YY & 36.81 & 4.57 & 9.11 & $\vee q \leq . \leq T$ & 29.10 & 27.30 & 2009 \\
\hline 2.98 & M.1 & 43.76 & 7.27 & 15.53 & 1707.11 & 31.66 & 52.31 & 2010 \\
\hline 3.16 & r^q.V & 71.52 & 5.44 & 14.87 & ד. & 51.84 & 35.36 & 2011 \\
\hline "2.44 & 258.42 & 40.65 & 6.18 & *11.36 & 1149.23 & 33.56 & 33.59 & 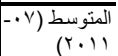 \\
\hline
\end{tabular}

المصدر: صندوق دعم صناعة الغزل و النسيج , الإدارة العامة للبحوث , النشرة الربع سنوية ـ أعداد متفرقة.

جدول رقم (؛) : معـادلات القيم الإتجاهيـة الزمنيـة لتطور كل مـن كميـة الصـادرات وسـعر التصـدير وقيمـة

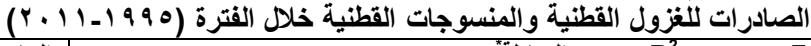

\begin{tabular}{|c|c|c|c|c|}
\hline البيان & المعادلة" & $\mathbf{R}^{2}$ & $\mathbf{F}$ & P-Value \\
\hline \multirow{3}{*}{ 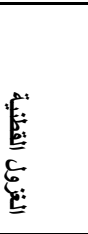 } & $\begin{aligned} \operatorname{Ln} 91= & \varepsilon 9.109-. . r Y X \\
& (8.440)(-2.731)\end{aligned}$ & 0.33 & 7.46 & 0.015 \\
\hline & $\begin{aligned} \operatorname{Ln} y_{2}= & 11.692+0.065 X \\
& (12.613)(8.463)\end{aligned}$ & 0.83 & 71.62 & 0.000 \\
\hline & 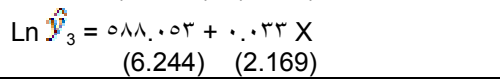 & 0.24 & 4.70 & 0.046 \\
\hline \multirow{3}{*}{ 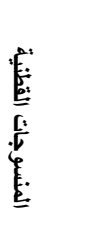 } & $\begin{aligned} \operatorname{Ln} \hat{y}_{:}= & 10.1 \mu r-. \cdot v \cdot X \\
& (6.912) \quad(-\varepsilon .99 \cdot)\end{aligned}$ & 0.62 & 24.90 & 0.000 \\
\hline & $\begin{aligned} \operatorname{Ln} y_{0}= & 11.444+0.0 \vee r X \\
& (8.812) \quad(7 . \wedge \vee r)\end{aligned}$ & $.1 \mathrm{VT}$ & $\varepsilon V, r \varepsilon$ & 0.000 \\
\hline & $y_{1}=377.821-59.966 x+3.423 x^{2}$ & 0.78 & 25.13 & 0.000 \\
\hline
\end{tabular}

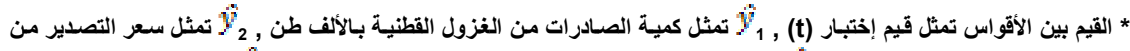

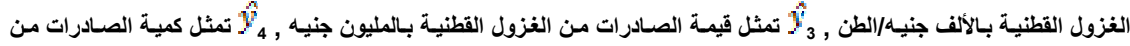

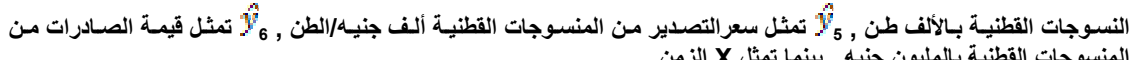

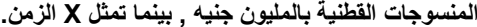

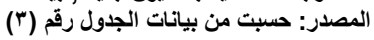


- تطور صادرات الملابس الجاهزة:

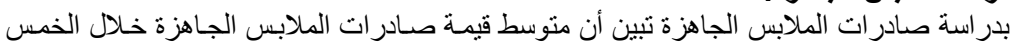

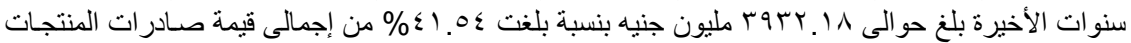

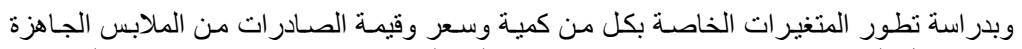

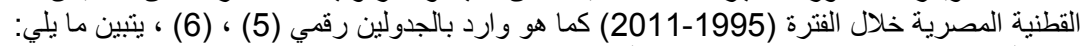

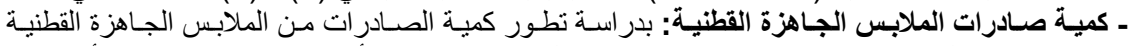

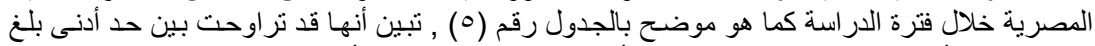

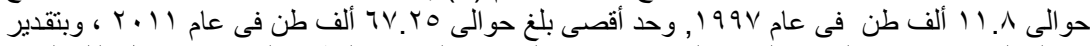

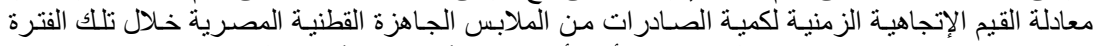

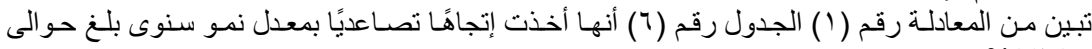
\%) Y.1.

ـ سعر التصدير الملابس الجـاهزة القطنيـة: بدراسـة تطور سعر التصدير الملابس الجـاهزة القطنية المصرية

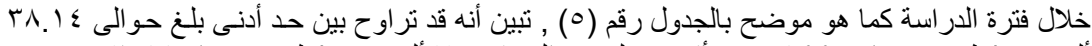

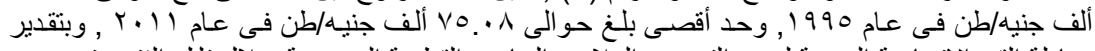

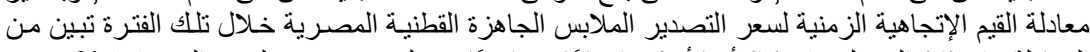

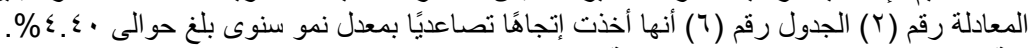

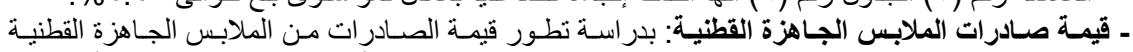

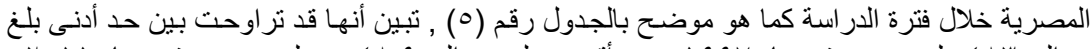

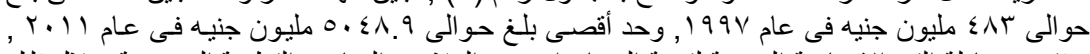

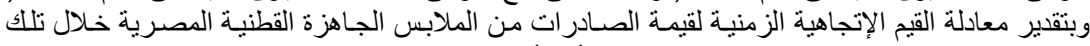

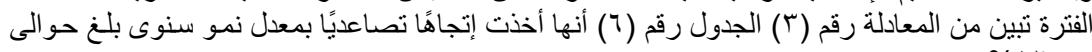

مما سبق يتضح أهمية صناعة الملابس الجـاهزة القطنية فى دعم الميزان التجارى المصرى نظرًا \%17.0.

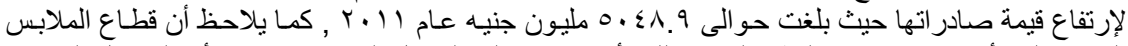

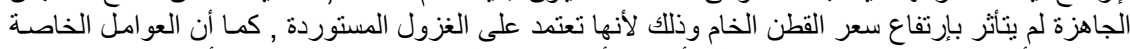

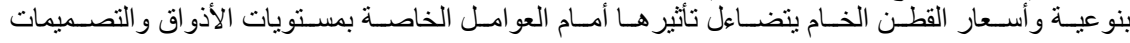
و التكنولوجيا المستخدمة في التصنئ التطنيع. ـ تطور صادرات التريكو القطنية:

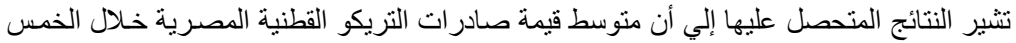

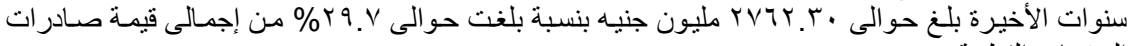
المنتجات القطنية. وبدر اسة تطور كل من كمية وسعر وقيمة الصسادرات من التريكو المصرية خـلال الفترة (1995-

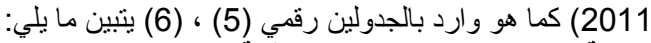

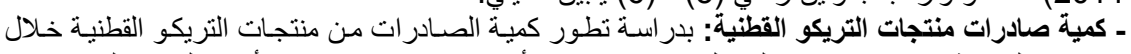

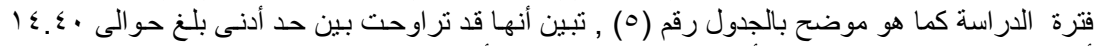

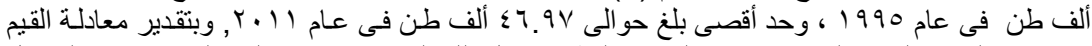

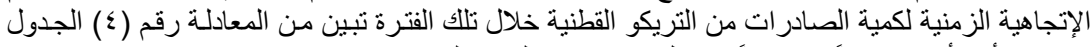

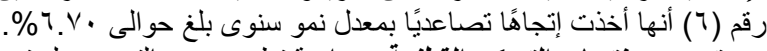

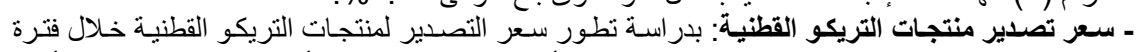

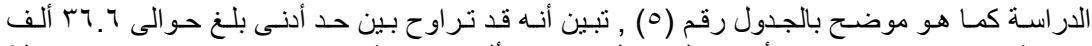

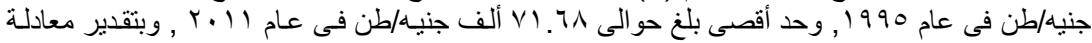

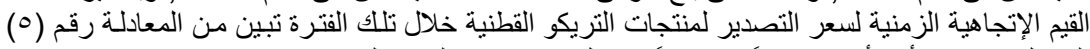

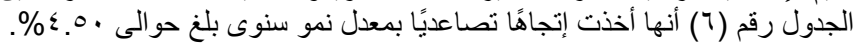

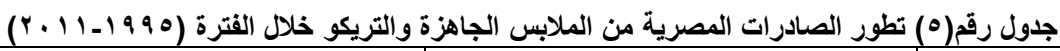
التزيكو

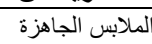




\begin{tabular}{|c|c|c|c|c|c|c|c|c|}
\hline قالنسة صادة لإجمالى المنات & (مليون جنيه) & (ألف جنيه) & (ألف طن) & قالنسبة صنادرات الإمالى & (مليون جنيه) & (ألف جنيه) & (ألف طن) & \\
\hline 20.60 & OrV.E & 36.60 & 14.40 & 19.20 & $\sum q r_{.} \cdot 1$ & 38.14 & 12.90 & 1995 \\
\hline 28.00 & $T \leq \varepsilon . T V$ & 37.70 & 17.10 & 22.70 & OrY.T. & 39.00 & 13.40 & 1996 \\
\hline 28.40 & VVr.el & 40.90 & 18.90 & 17.80 & $\varepsilon \wedge r .7 r$ & 40.90 & 11.80 & 1997 \\
\hline 34.60 & $\wedge 9 ., r \leq$ & 41.60 & 21.40 & 20.40 & oro.r & 39.20 & 13.40 & 1998 \\
\hline 39.10 & $\wedge q . . \leqslant \varepsilon$ & 45.20 & 19.70 & 24.60 & $071 . .$. & 42.50 & 13.20 & 1999 \\
\hline 35.70 & ו..r. & 44.20 & 22.70 & 27.80 & vAr... & 42.50 & 18.40 & 2000 \\
\hline 34.40 & $1 \cdots 1 . \wedge \Lambda$ & 48.40 & 20.70 & 30.30 & אי.YNA & 49.90 & 17.70 & 2001 \\
\hline 36.20 & 1178.90 & 49.70 & 23.50 & 29.40 & $9 \leqslant 9.70$ & 48.70 & 19.50 & 2002 \\
\hline 36.40 & IIVT.qT & 49.70 & 23.60 & 29.40 & $9 \leq 9.70$ & 48.70 & 19.50 & 2003 \\
\hline 38.10 & $r \cdot r \cdot . I r$ & 65.70 & 30.90 & 29.00 & $10 \leqslant \varepsilon . V 7$ & 61.30 & 25.20 & 2004 \\
\hline 35.10 & MrE... & 60.00 & 30.40 & 32.00 & $177 \varepsilon .7$. & 57.40 & 29.00 & 2005 \\
\hline 31.60 & r).0.7. & 65.80 & 32.00 & 39.00 & roq^. $\leqslant \varepsilon$ & 49.40 & 52.60 & 2006 \\
\hline 33.10 & rrq. rی & 64.70 & 35.40 & 39.80 & $r V \leqslant \Lambda . r$. & 60.40 & 45.50 & 2007 \\
\hline 31.08 & rTVT.YK & 64.26 & 41.60 & 40.76 & ro.0.71 & 64.86 & 54.05 & 2008 \\
\hline 31.58 & rvo0.11 & 69.98 & 39.37 & 42.88 & rvE. . Tr & 72.96 & 51.27 & 2009 \\
\hline 25.58 & TVYV.TV & 69.02 & 39.52 & 43.28 & $\leq 7) \leq . \wedge \wedge$ & 69.88 & 66.04 & 2010 \\
\hline 27.31 & ון. דרזr & 71.68 & 46.97 & 40.96 & $0.59 .1 T$ & 75.08 & 67.25 & 2011 \\
\hline *29.73 & 2762.30 & 67.93 & 40.57 & ${ }^{*} 41.54$ & 3932.18 & 68.64 & 56.82 & 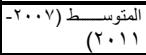 \\
\hline
\end{tabular}

المصدر: صندوق دعم صناعة الغزل و النسيج , الإدارة العامة للبحوث , النشرة الربع سنوية , أعداد متفرقة.

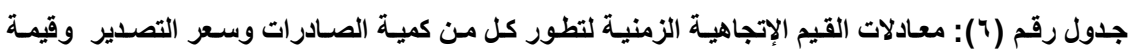

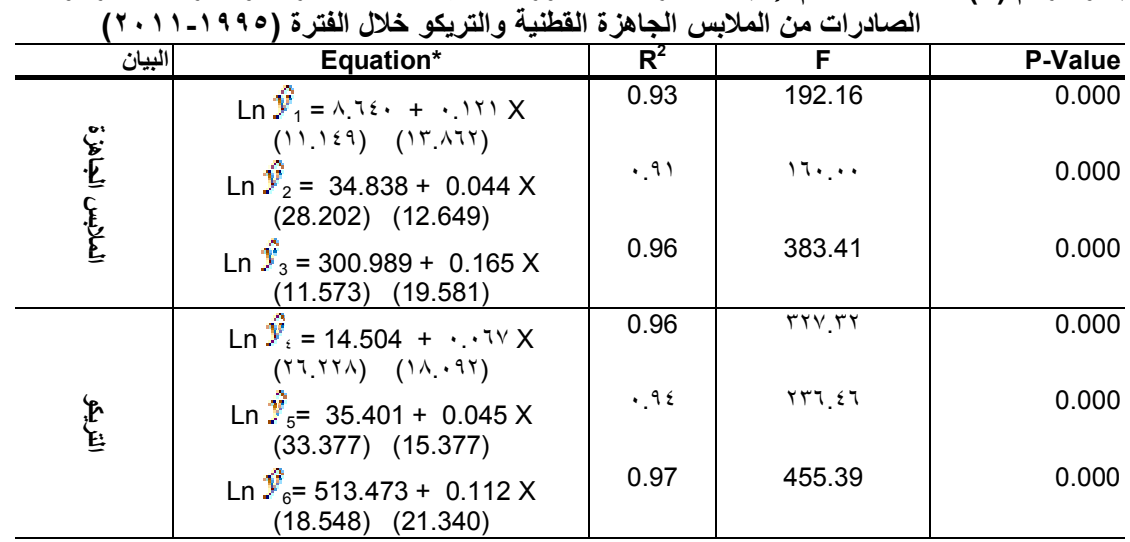

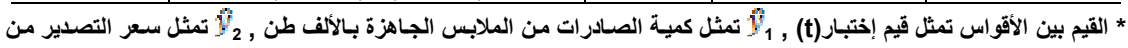

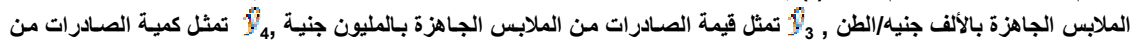

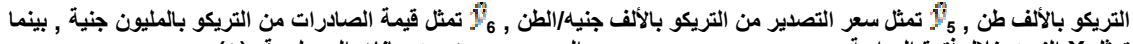
المصدر: حسبت من بيانات الجدول رقم (ه).

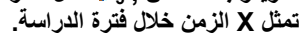

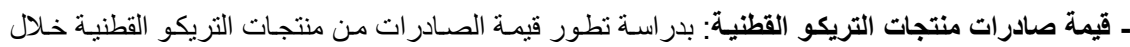

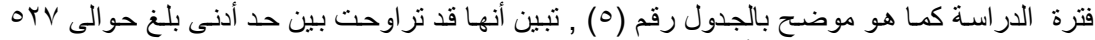

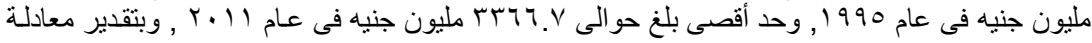




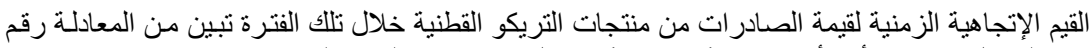

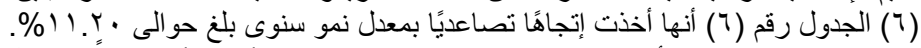

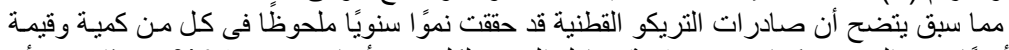

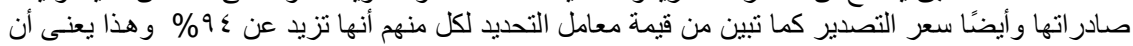

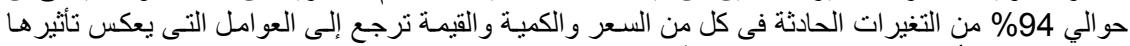

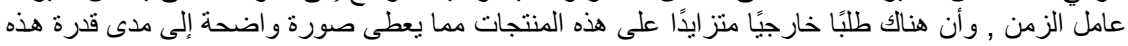

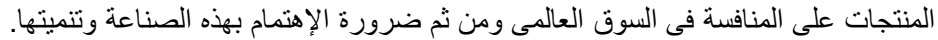
ـ تطور صادرات المفصلات القطنية:

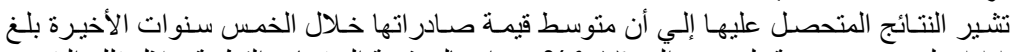

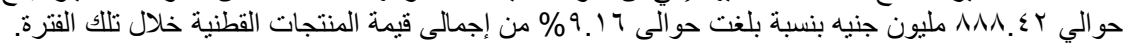

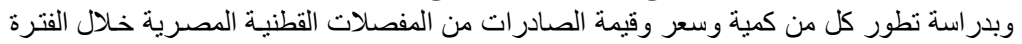

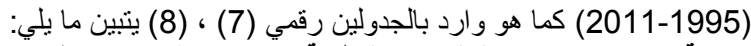

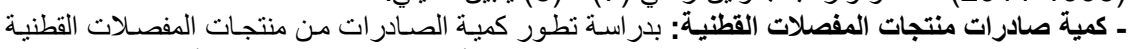

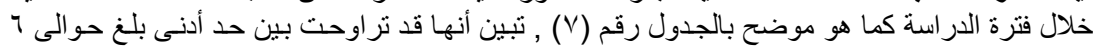

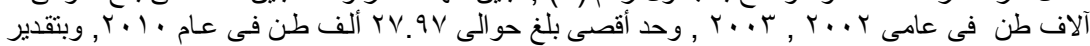

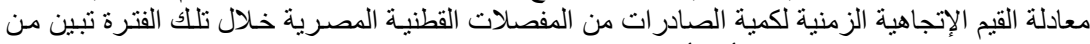

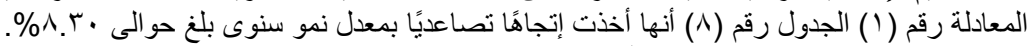

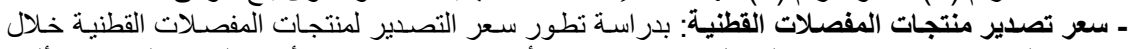

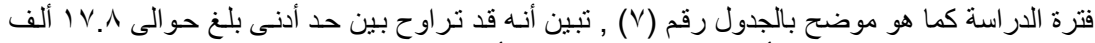

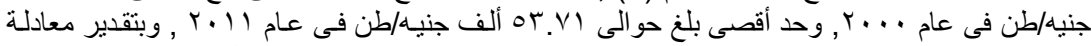

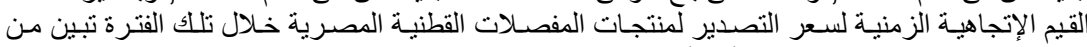

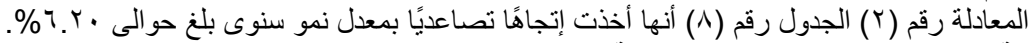

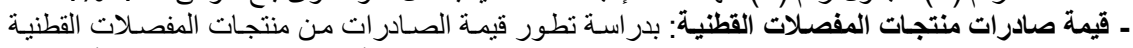

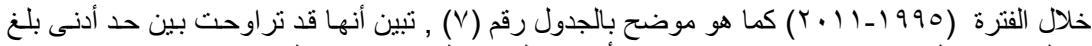

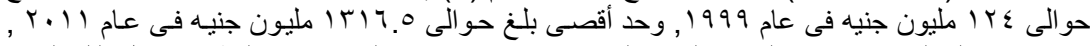

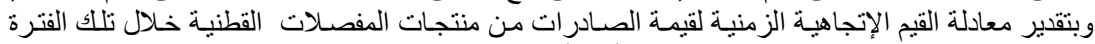

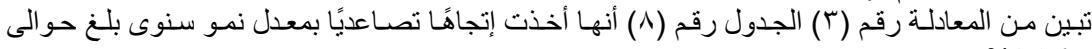
$\%$ \% 1 . 9.

ـ تطور صادرات المنتجات الوبرية القطنية:

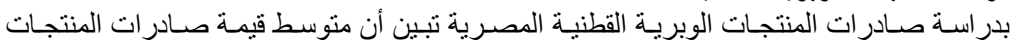

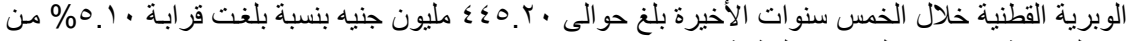
إجمالى قيمة صادر ات المنتجات الفنات القطنية .

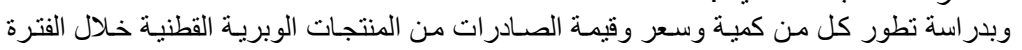

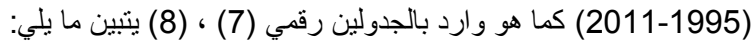

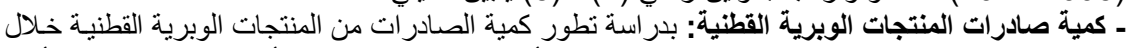

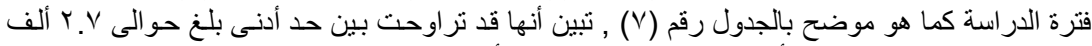

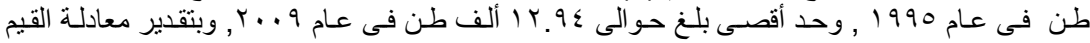

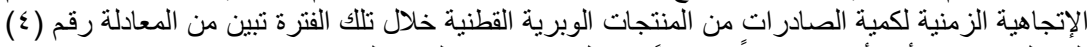

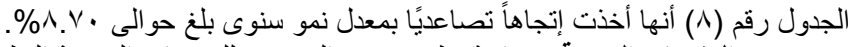

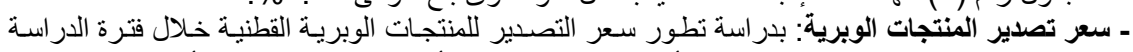

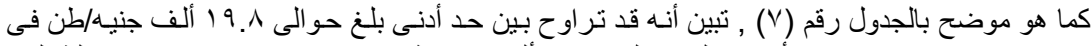

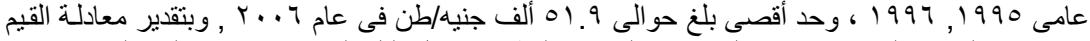

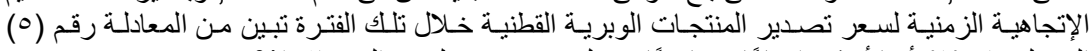

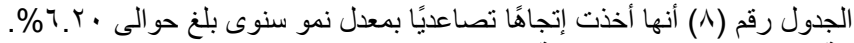

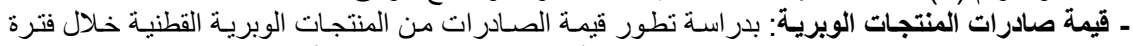

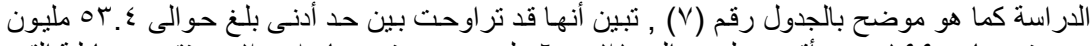

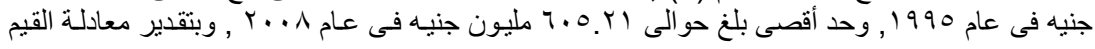


J. Agric. Econom. and Social Sci., Mansoura Univ., Vol.5 (10), October, 2014

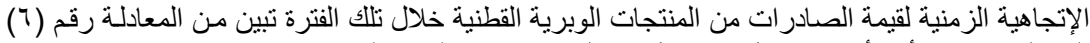

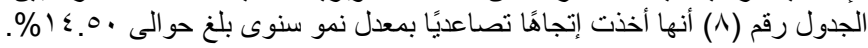

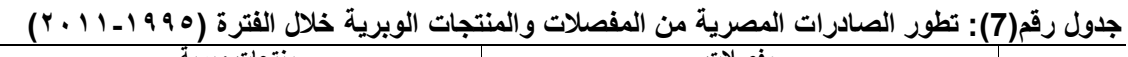

\begin{tabular}{|c|c|c|c|c|c|c|c|c|}
\hline \multicolumn{4}{|c|}{ منتجات وبرية } & \multicolumn{4}{|c|}{ مفصلات } & \multirow[b]{2}{*}{ السنة } \\
\hline لإجمالى قيمة المنتجات & (مليون جنيه) & (ألف جنيه) & (ألف طُن) & 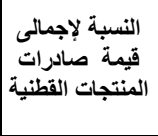 & (مليون جنيه) & (ألف جنيه) & (ألف طنّ) & \\
\hline 2.10 & or. $\leqslant 4$ & 19.80 & 2.70 & 6.30 & 171.1. & 17.90 & 9.00 & 1995 \\
\hline 2.40 & $00 . \leqslant \varepsilon$ & 19.80 & 2.80 & 7.00 & 171.99 & 18.10 & 8.90 & 1996 \\
\hline 2.80 & V.. 41 & 21.30 & 3.60 & 5.20 & $1 \leqslant 1 . V_{0}$ & 18.90 & 7.50 & 1997 \\
\hline 3.90 & $99 . \leqslant 0$ & 22.10 & 4.50 & 5.50 & $1 \leqslant 1 .$. & 18.80 & 7.50 & 1998 \\
\hline 5.20 & $\| \wedge . \wedge$. & 21.60 & 5.50 & 5.40 & $1 K r .90$ & 18.50 & 6.70 & 1999 \\
\hline 4.00 & $111 . \wedge r$ & 21.10 & 5.30 & 4.90 & $1 T V . T$ & 17.80 & 7.70 & 2000 \\
\hline 5.00 & $101 . .1$ & 23.60 & 6.40 & 5.10 & $1 \leqslant v .71$ & 20.80 & 7.10 & 2001 \\
\hline 6.50 & $r 1 . .17$ & 29.60 & 7.10 & 4.40 & $1 \leqslant 1 .$. & 23.50 & 6.00 & 2002 \\
\hline 6.50 & $r 1 . .17$ & 29.60 & 7.10 & 4.40 & $1 \leqslant 1.7$. & 23.60 & 6.00 & 2003 \\
\hline 6.40 & $r \mu q . \varepsilon r$ & 37.30 & 9.10 & 7.40 & एवा. & 36.10 & 10.90 & 2004 \\
\hline 7.70 & ए१९. ८१ & 43.90 & 9.10 & 7.40 & 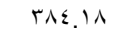 & 33.70 & 11.40 & 2005 \\
\hline 7.30 & ENY.TV & 51.90 & 9.30 & 6.90 & $\varepsilon 0 \wedge, Y \leqslant$ & 35.80 & 12.80 & 2006 \\
\hline 8.20 & 071.19 & 44.10 & 12.90 & 5.90 & $\varepsilon .0 .9 Y$ & 34.40 & 11.80 & 2007 \\
\hline 7.04 & 7.0 .4$. & 46.77 & 12.94 & 9.45 & 110.91 & 31.18 & 26.17 & 2008 \\
\hline 4.06 & ros. $\cdot V$ & 34.51 & 10.26 & 10.32 & $9 \ldots . \leqslant 9$ & 32.38 & 27.81 & 2009 \\
\hline 3.15 & Tro. $\varepsilon V$ & 36.15 & 9.28 & 9.41 & 1..r.rA & 35.87 & 27.97 & 2010 \\
\hline 2.94 & MTY.rV & 50.26 & 7.21 & 10.68 & $1 \pi 17.2 \pi$ & 53.71 & 24.51 & 2011 \\
\hline 5.08 & 445.20 & 42.36 & 10.52 & 9.16 & 888.42 & 37.51 & 23.65 & متوسط (Y) (Y) \\
\hline
\end{tabular}


Elshahawy, M. I. M. et al.

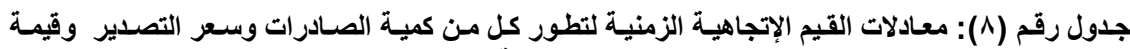

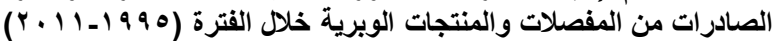

\begin{tabular}{|c|c|c|c|c|}
\hline & Equation & $\mathbf{R}^{2}$ & $\mathbf{F}$ & P-Value \\
\hline \multirow{3}{*}{ E. } & 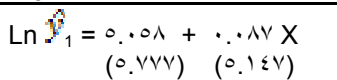 & 0.64 & rฯ. $\varepsilon 9$ & 0.000 \\
\hline & $\begin{aligned} \operatorname{Ln} \hat{y}_{2}= & 14.979+0.062 X \\
& (13.892)(8.830)\end{aligned}$ & $\cdot . \wedge \varepsilon$ & VV. 91 & 0.000 \\
\hline & $\begin{aligned} \operatorname{Ln} \hat{y}_{3}= & v 0 . v 4 \Lambda+0.149 X \\
& (0 . r \leq 0)(\Lambda . \cdots v)\end{aligned}$ & 0.81 & 64.10 & 0.000 \\
\hline \multirow{3}{*}{ 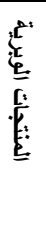 } & 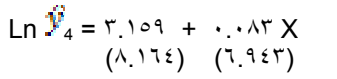 & $\cdot v^{17}$ & $\varepsilon \wedge, Y$. & 0.000 \\
\hline & $\begin{aligned} \operatorname{Ln} \hat{y}_{5}= & 17.555+0.062 X \\
& (11.749) \quad(7.477)\end{aligned}$ & $\cdot v^{19}$ & 00.9. & 0.000 \\
\hline & $\begin{aligned} & \operatorname{Ln} y_{6}= 55.459+0.145 X \\
&(0 . \wedge v \varepsilon)(8.732)\end{aligned}$ & 0.84 & 76.25 & 0.000 \\
\hline
\end{tabular}

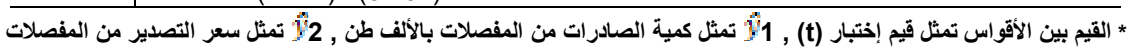

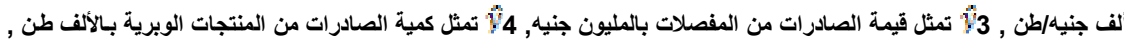

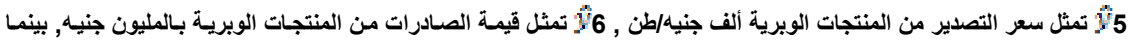

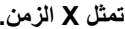
المصدر: حسبت من بيانات الجدول رقم (V) المنر). -تطور صادرات القطن الطبى:

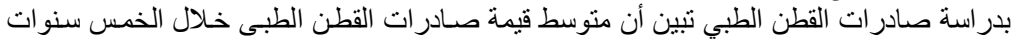

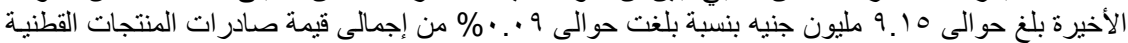
المصرية. وبدر اسـة تطور كل مـن كميـة وسـر وقيمـة الصـادرات مـن القطن الطبي خـلال الفترة (1995-

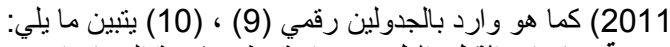

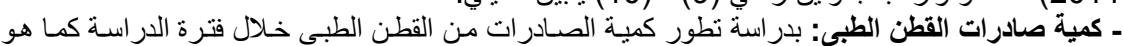

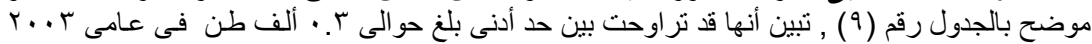

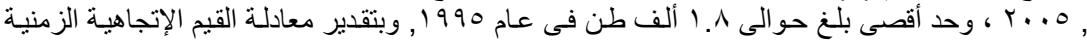

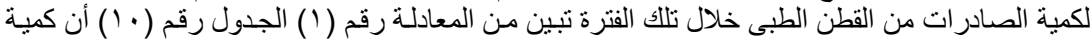

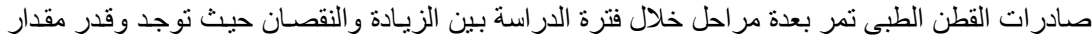

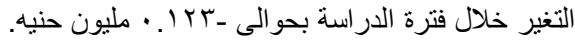

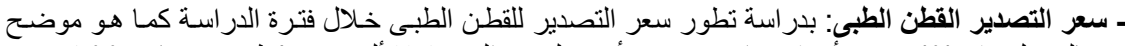

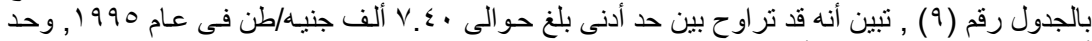

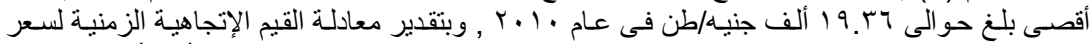

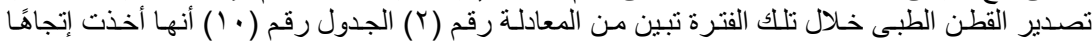

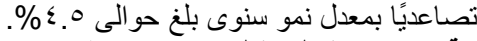

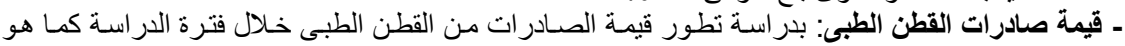

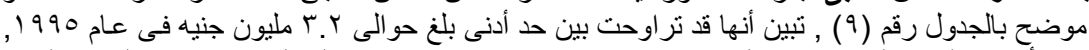

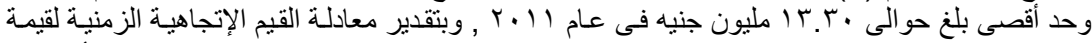

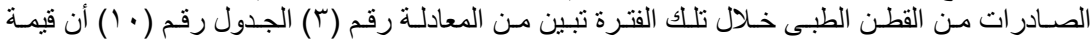

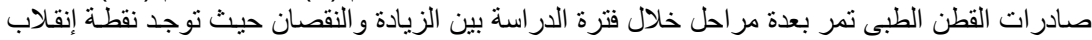

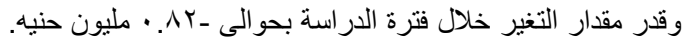

جدول رقم(9): تطور الصادرات المصرية من القطن الطبى وإجمالى صـادرات المنتجات القطنية خلال الفترة (99 9 (1 (r.) (1)

السنة




\begin{tabular}{|c|c|c|c|c|c|c|c|c|}
\hline قيمة صنادة لإجماتى & (مليون جنيه) & (ألف جنيه) & (ألف طنّة) & المنيمة صادة لاجمالى النسات & (مليون جنيه) & (ألف جنيه) & (ألف طنّة) & \\
\hline 100 & 2563.00 & 20.77 & 123.40 & 0.50 & 13.30 & 7.40 & 1.80 & 1995 \\
\hline 100 & 2303.90 & 22.72 & 101.40 & 0.30 & 6.90 & 8.60 & 0.80 & 1996 \\
\hline 100 & 2719.60 & 21.90 & 124.20 & 0.20 & 5.50 & 9.20 & 0.60 & 1997 \\
\hline 100 & 2577.70 & 24.71 & 104.30 & 0.10 & 5.00 & 10.00 & 0.50 & 1998 \\
\hline 100 & 2277.00 & 27.01 & 84.30 & 0.10 & 3.20 & 8.00 & 0.40 & 1999 \\
\hline 100 & 2811.50 & 26.96 & 104.30 & 0.20 & 3.30 & 8.30 & 0.40 & 2000 \\
\hline 100 & 2913.10 & 30.38 & 95.90 & 0.10 & 4.40 & 8.80 & 0.50 & 2001 \\
\hline 100 & 3227.50 & 32.54 & 99.20 & 0.10 & 3.80 & 9.50 & 0.40 & 2002 \\
\hline 100 & 3227.50 & 32.57 & 99.10 & 0.10 & 3.80 & 12.70 & 0.30 & 2003 \\
\hline 100 & 5336.00 & 46.44 & 114.90 & 0.10 & 5.40 & 13.50 & 0.40 & 2004 \\
\hline 100 & 5195.30 & 44.44 & 116.90 & 0.10 & 5.10 & 17.00 & 0.30 & 2005 \\
\hline 100 & 6655.30 & 46.61 & 142.80 & 0.10 & 6.30 & 12.60 & 0.50 & 2006 \\
\hline 100 & 6909.00 & 49.78 & 138.80 & 0.10 & 7.50 & 15.00 & 0.50 & 2007 \\
\hline 100 & 8600.17 & 50.79 & 169.34 & 0.09 & 8.12 & 10.68 & 0.76 & 2008 \\
\hline 100 & 8722.95 & 54.05 & 161.39 & 0.12 & 10.04 & 12.55 & 0.80 & 2009 \\
\hline 100 & 10664.50 & 52.57 & 202.85 & 0.09 & 9.10 & 19.36 & 0.47 & 2010 \\
\hline 100 & 12327.60 & 65.73 & 187.56 & 0.09 & 10.98 & 13.73 & 0.80 & 2011 \\
\hline 100 & 5237.15 & 38.23 & 127.68 & 0.09 & 9.15 & 14.26 & 0.66 & 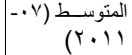 \\
\hline \multicolumn{9}{|c|}{ 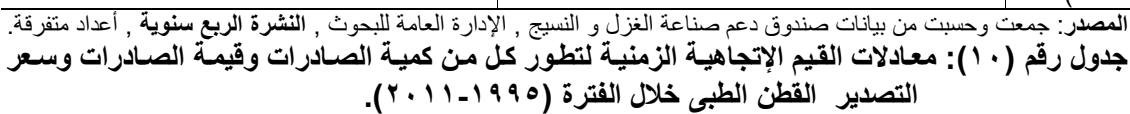 } \\
\hline No. & \multicolumn{4}{|c|}{ Equation } & $\mathrm{R}^{2}$ & & P-Value \\
\hline 1 & \multicolumn{4}{|c|}{$\begin{array}{c}\hat{P}_{1}=1.440-0.231 X+0.012 X^{2} \\
\quad(7.496) \quad(-4.699) \quad(4.449)\end{array}$} & .710 & \multicolumn{2}{|c|}{11.170} & 0.00 \\
\hline r & \multicolumn{4}{|c|}{$\begin{array}{c}\operatorname{Ln} y_{2}=17.555+0.045 x \\
(11.448)(5.248)\end{array}$} & $.7 \leqslant V$ & \multicolumn{2}{|c|}{ rV.OS. } & 0.00 \\
\hline r & \multicolumn{4}{|c|}{$\begin{array}{c}f_{3}=11.292-1.801 X+0.109 X^{2} \\
(9.168)(-5.718)(6.435)\end{array}$} & 0.769 & \multicolumn{2}{|c|}{23.295} & 0.00 \\
\hline
\end{tabular}

* القيم بين الأقواس تمثل قيم إختبار (t) , $\hat{l}_{1}$ تمثل كمية الصادرات من القطن الطبى بالالف طن , الطبى الف جنيه/الطن , 3 ر تمثل قيمة الصادرات الإجمالية من القطن الطبى بالمليون جنية, بينما تمثل X

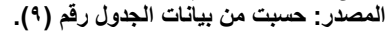

الأهمية النسبية لتأثير كلا من سعر التصدير وكمية الصادرات علي قيمة صادرات أهم المنتجات القطنية: - بالنسبة لقيمة صادرات الفزول القطنية:

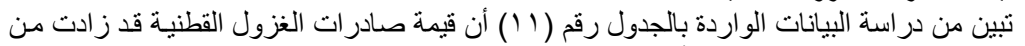

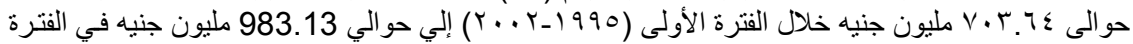

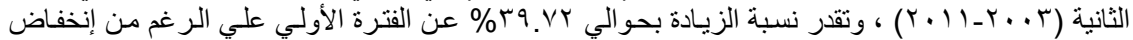

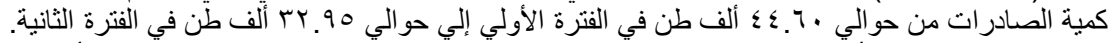

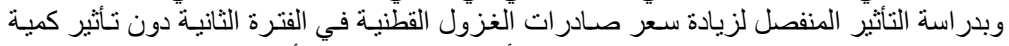

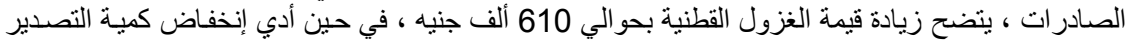

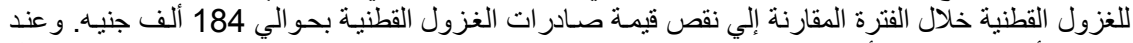

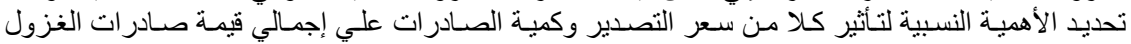

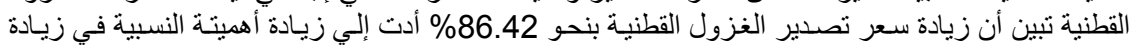

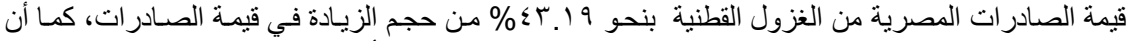

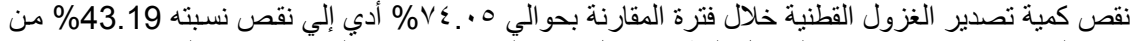

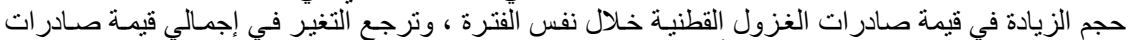

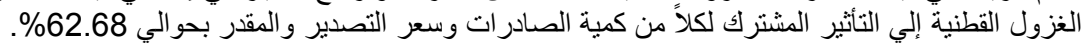


Elshahawy, M. I. M. et al.

جدول رقم (1'): أثر كمية الصادرات وسعر التصدير علي التغير في قيمة صادرات الغزول القطنية المصرية

\begin{tabular}{|c|c|c|c|c|c|c|c|}
\hline \multirow{2}{*}{ 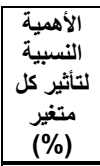 } & \multicolumn{3}{|c|}{ الكمية والثر التغير فين فترتي من الدراسة } & \multicolumn{3}{|c|}{ متوسط فترتى الدراسة } & \multirow[b]{2}{*}{ اليبان } \\
\hline & |لتأثتير المتتابع & المشترك لكل & المنفصل لكل & $\begin{array}{c}\text { × الرقم القياسنى } 100 \\
100 \\
100\end{array}$ & $\begin{array}{c}(2003-2011) \\
(2)\end{array}$ & $\begin{array}{c}(1995-2002) \\
(1)\end{array}$ & \\
\hline-43.19 & - & - & -0.184 & 74.05 & 32.95 & 44.60 & 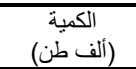 \\
\hline 143.19 & - & - & 0.610 & 186.42 & 29.51 & 15.83 & (ألف جنيد/طن) \\
\hline 100 & 0.267 & -0.159 & 0.426 & 139.72 & 983.13 & 703.64 & (مليون جنبة) \\
\hline
\end{tabular}

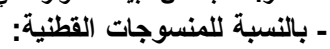

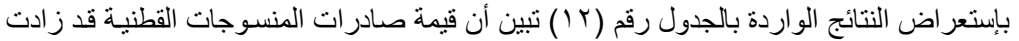

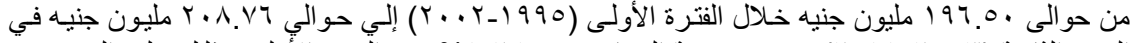

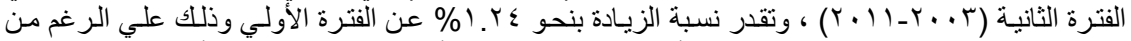

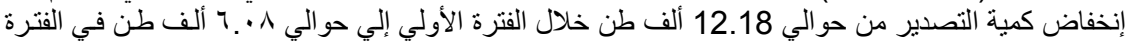

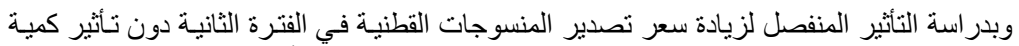

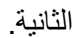

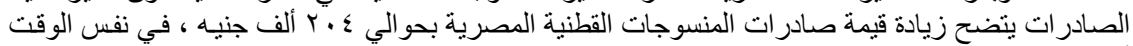

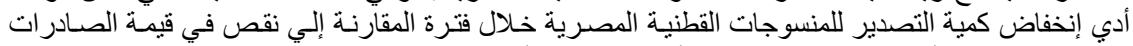

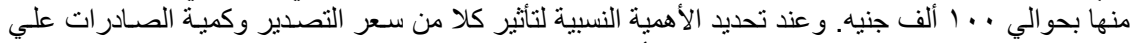

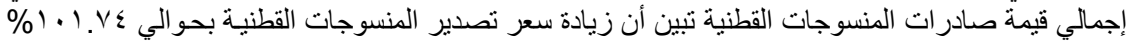

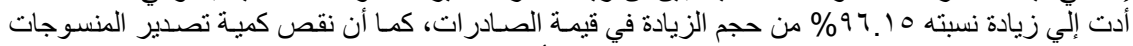

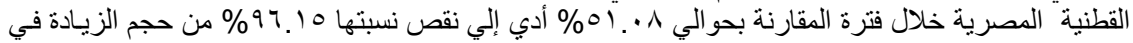

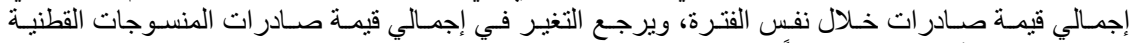
المصرية إلي التأثثر المشترك لكلاً من كمية الصادرات وسعر التصدير والمقدر بحوالي 1.92\% 
جدول رقم (r 1 ): أثر كمية الصـادرات وسـر التصدير علي التغير في قيمـة صـادرات المنسوجات القطنية

\begin{tabular}{|c|c|c|c|c|c|c|c|}
\hline \multirow{2}{*}{ 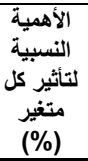 } & \multicolumn{3}{|c|}{ 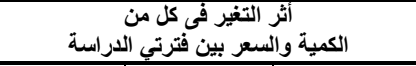 } & \multicolumn{3}{|c|}{ متوسط فترنى الاراسة } & \multirow[b]{2}{*}{ اليبان } \\
\hline & اللتأثير المتتابع & الكل المتغيرين المشترك & الكلثير متغير المنصل & 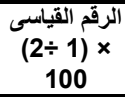 & $\begin{array}{c}2003- \\
2011) \\
(2)\end{array}$ & $\begin{array}{c}(1995-2002) \\
(1)\end{array}$ & \\
\hline-96.15 & - & & -0.100 & 49.92 & 6.08 & 12.18 & (الفتِّنة) \\
\hline 196.15 & - & & 0.204 & 201.74 & 33.16 & 16.44 & (ألف جنيه/طن) \\
\hline 100 & 0.002 & -0.102 & 0.104 & 101.24 & 208.76 & 196.50 & |(مليون جنيه) \\
\hline
\end{tabular}

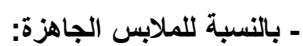

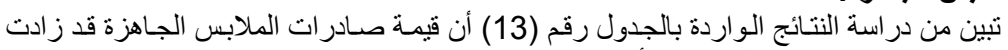

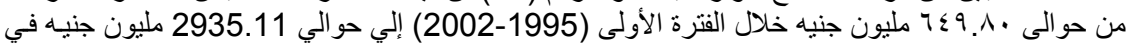

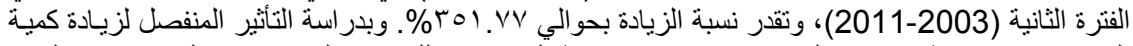

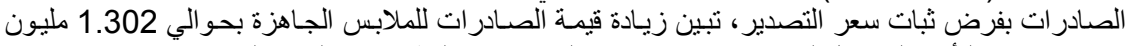

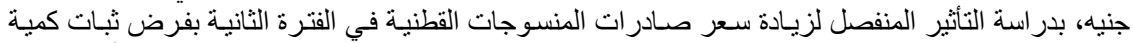

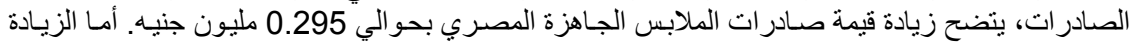

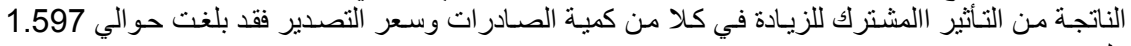

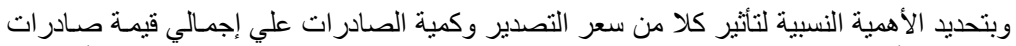
مليون جنيه.

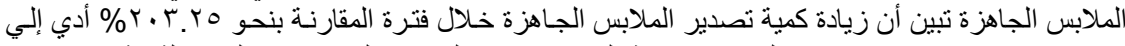

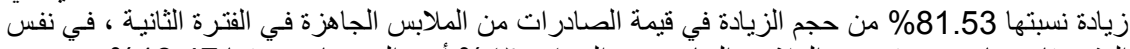

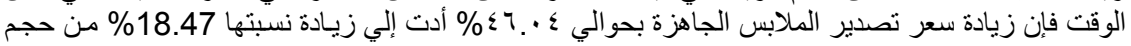

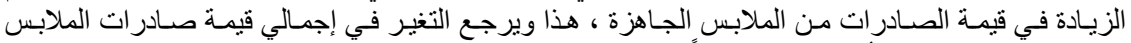
الجاهزة المصري إلي التأثثر المشترك لكلاً من كمية الصادرات وسعر التصدير والتهي والمقدر بحوالي 137.57\%.

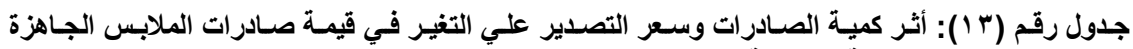

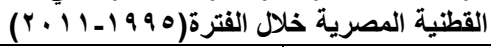

\begin{tabular}{|c|c|c|c|c|c|c|c|}
\hline \multirow[b]{2}{*}{ الأهمية النسبية لتأثير كل } & \multicolumn{3}{|c|}{ الكمية والثُر التفير في كل منتي الدراسة } & \multicolumn{3}{|c|}{ متوسط فترتى الاراسة } & \multirow[b]{2}{*}{ اليبان } \\
\hline & التأثتير المتتابع & |التاثير المشترك لكل & التأثير متغنفير & 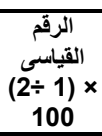 & $\begin{array}{c}(2003- \\
2011) \\
(2)\end{array}$ & $\begin{array}{c}(1995- \\
2002) \\
(1)\end{array}$ & \\
\hline 81.53 & - & - & 1.302 & 303.25 & 45.60 & 15.04 & (ألف طنية) \\
\hline 18.47 & - & - & 0.295 & 146.04 & 62.22 & 42.61 & جنيه/طن) \\
\hline 100 & 2.197 & 0.600 & 1.597 & 451.77 & 2935.11 & 649.80 & $\begin{array}{l}\text { (مليون } \\
\text { (القيمة }\end{array}$ \\
\hline
\end{tabular}

- بالنسبة للتريكو: 


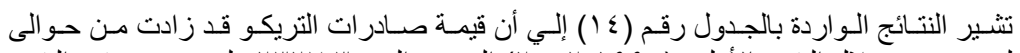

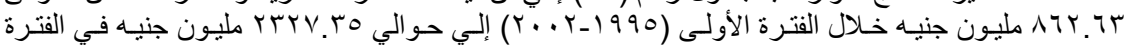

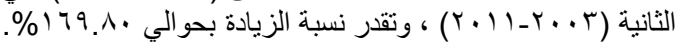

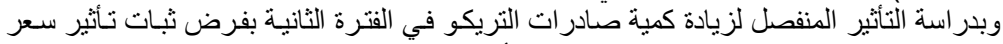

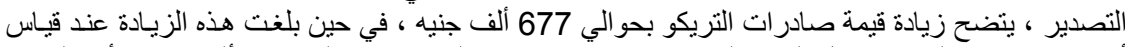

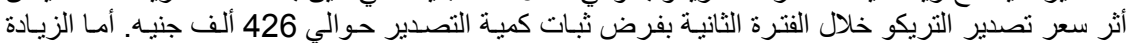

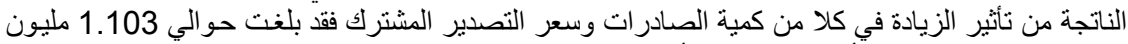

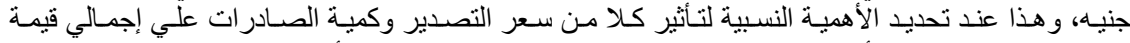

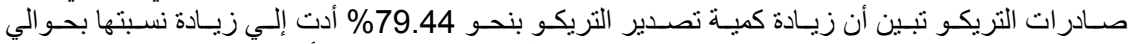

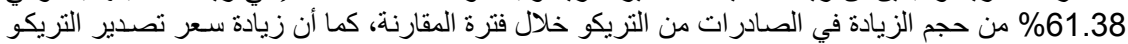

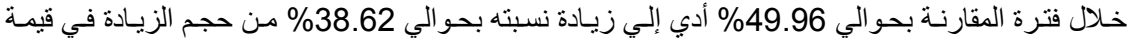

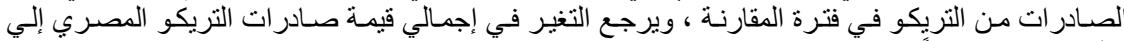

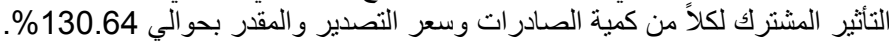

جدول رقم (ع 1): أثر كمية الصادرات وسعر التصدير علي التغير في قيمة صادرات التريكو القطنية المصرية

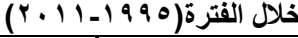

\begin{tabular}{|c|c|c|c|c|c|c|c|}
\hline \multirow[b]{2}{*}{ الأهمية النسبية لتأثير كل } & \multicolumn{3}{|c|}{ 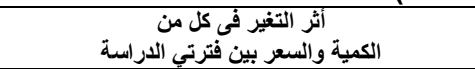 } & \multicolumn{3}{|c|}{ متوسط فترتى الدراسة } & \multirow[b]{2}{*}{ اليبان } \\
\hline & اللتأثتير المتتابع & التأثير المشترك لكل & التكل متغير المنفل & 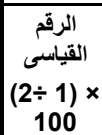 & \begin{tabular}{|c|}
$(2003-$ \\
$2011)$ \\
$(2)$
\end{tabular} & $\begin{array}{c}(1995- \\
2002) \\
(1)\end{array}$ & \\
\hline 61.38 & - & - & 0.677 & 179.44 & 35.53 & 19.80 & \begin{tabular}{|l} 
(ألف طن) \\
(الكبة)
\end{tabular} \\
\hline 38.62 & - & - & 0.426 & 149.96 & 64.54 & 43.04 & جنيه/طن) \\
\hline 100 & 1.441 & 0.338 & 1.103 & 269.89 & 2327.32 & 862.32 & (مليونة \\
\hline
\end{tabular}

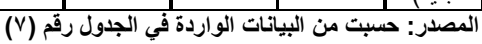

- مالنسبة للمفصلات القطنية:

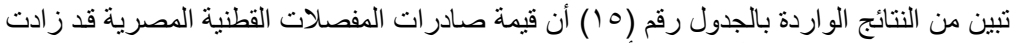

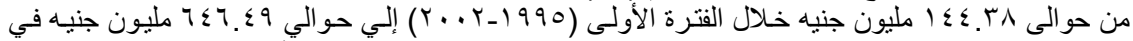

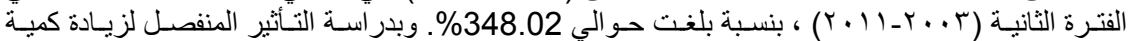

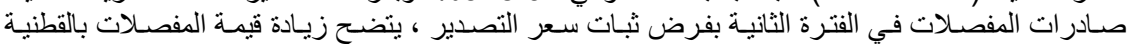

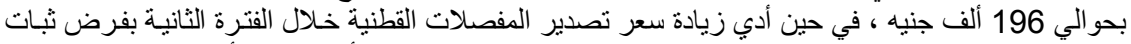

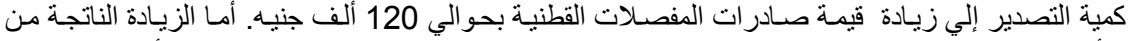

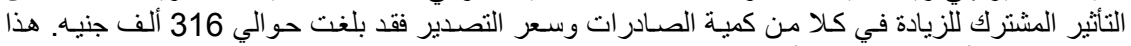

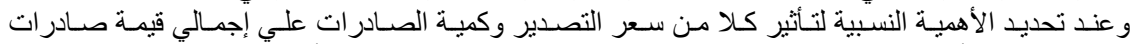

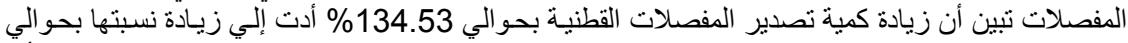

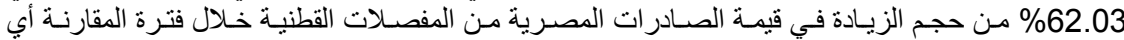

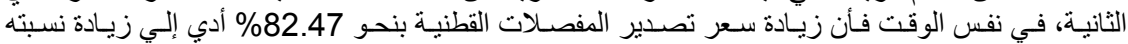

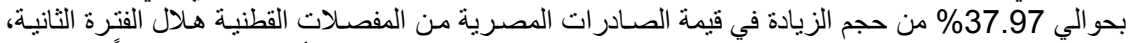

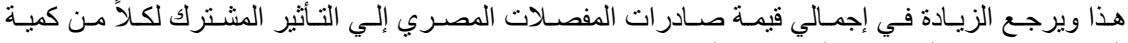

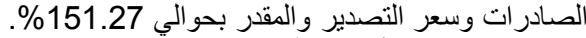

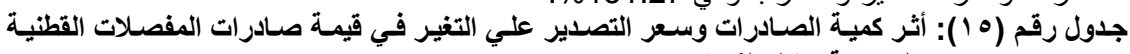

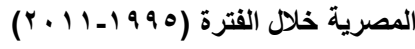




\begin{tabular}{|c|c|c|c|c|c|c|c|}
\hline \multirow[b]{2}{*}{ الأهمية النسبية لتأثير كل } & \multicolumn{3}{|c|}{ الكمية والثر التغير في كترتي من الدراسة } & \multicolumn{3}{|c|}{ متوسط فترتى الدراسة } & \multirow[b]{2}{*}{ اليبان } \\
\hline & التأثير المتتابع & التأثير المشترك لكل & التأثلير متغنفير & 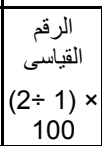 & $\begin{array}{c}(2003- \\
2011) \\
(2)\end{array}$ & $\begin{array}{c}(1995- \\
2002) \\
(1)\end{array}$ & \\
\hline 62.03 & - & - & 0.196 & 234.53 & 17.71 & 7.55 & (ألف طن) \\
\hline 37.97 & - & - & 0.120 & 182.47 & 35.19 & 19.29 & جنيه/طن) \\
\hline 100 & 0.478 & 0.162 & 0.316 & 448.02 & 646.62 & 144.33 & (مليونة \\
\hline
\end{tabular}

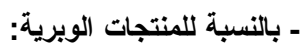

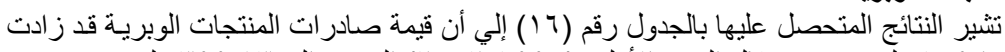

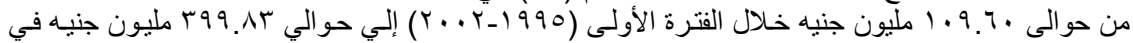

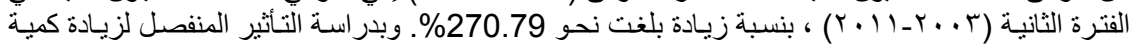

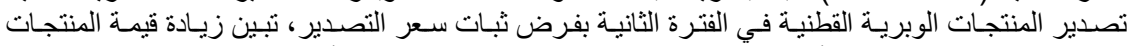

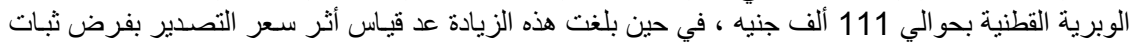

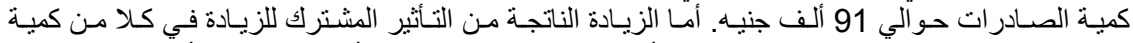

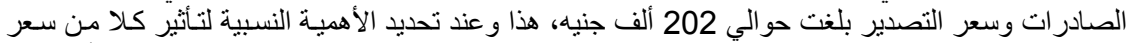

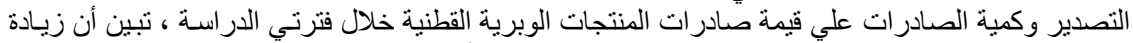

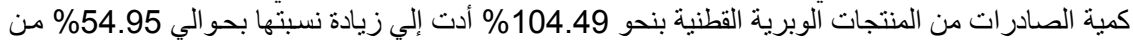

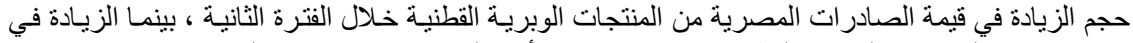

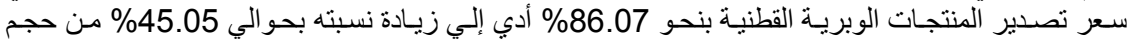

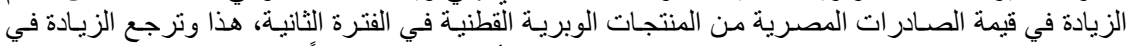

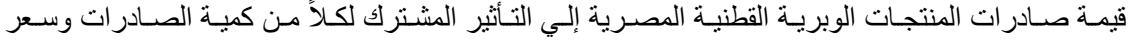
التصدير و المقدر بحوالي 147.03 \%

جدول رقم (1 1 ): أثر كميـة الصـادرات وسـعر التصدير علـي التغيـر في قيمـة صـادرات المنتجـات الوبريـة

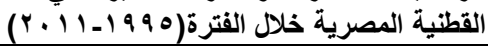

\begin{tabular}{|c|c|c|c|c|c|c|c|}
\hline \multirow{2}{*}{ الأهمية النسبية التأثير } & \multicolumn{3}{|c|}{ الكمية والثر التغر بين فتى كل من الدراسة } & \multicolumn{3}{|c|}{ متوسط فترتى الدراسة } & \multirow[b]{2}{*}{ اليبان } \\
\hline & اللتتابع التأثير & لكل المتغيرين التأثير & الكل متغير التأثير & $\begin{array}{c}\text { الرقم القياسى (1) } 1 \text { (100) } \\
\text { (100) }\end{array}$ & $\begin{array}{c}(2003- \\
2011) \\
(2)\end{array}$ & $\begin{array}{c}(1995- \\
2002) \\
(1)\end{array}$ & \\
\hline 54.95 & - & - & 0.111 & 204.49 & 9.69 & 4.74 & (ألفن طن) \\
\hline 45.05 & - & - & 0.091 & 186.07 & 41.61 & 22.36 & 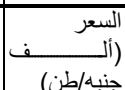 \\
\hline 100 & 0.297 & 0.095 & 0.202 & 370.79 & 406.42 & 109.61 & (مليون جنيه) \\
\hline
\end{tabular}

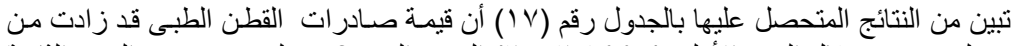

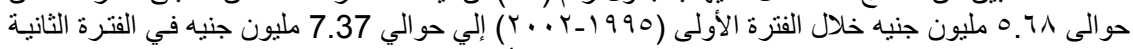

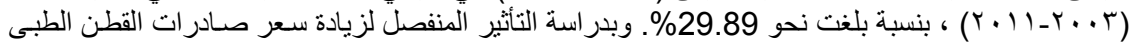




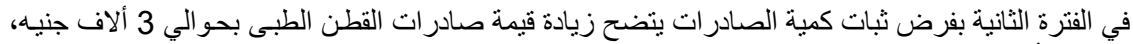

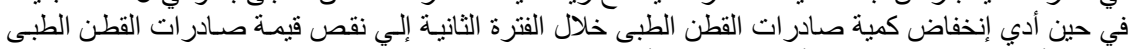

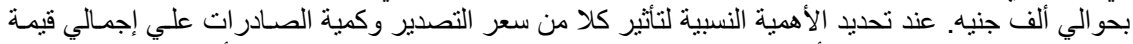

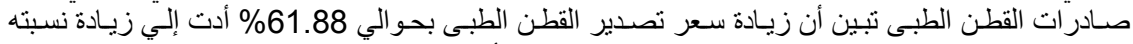

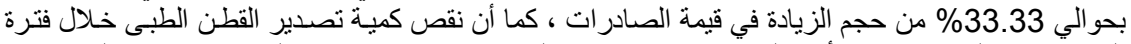

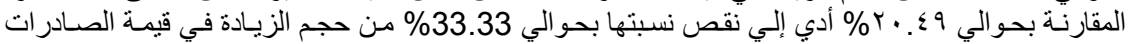

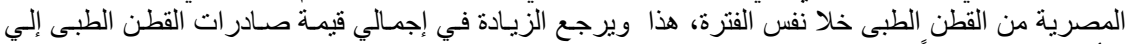

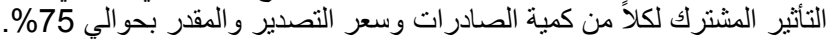

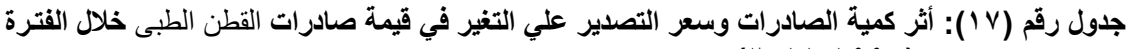
$(r+11-1990)$

\begin{tabular}{|c|c|c|c|c|c|c|c|}
\hline \multirow{2}{*}{ 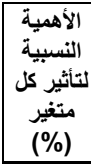 } & \multicolumn{3}{|c|}{ الكمية وألتر التغر بين فتى كل مني الدراسة } & \multicolumn{3}{|c|}{ متوسط فترتى الدراسة } & \multirow[b]{2}{*}{ اليبان } \\
\hline & اللمتغيرينيع & المشترك لكل التّير & المنفصل لكل & 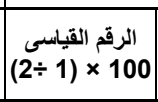 & $\begin{array}{c}(2003-2011) \\
(2)\end{array}$ & $\begin{array}{c}(1995-2002) \\
(1)\end{array}$ & \\
\hline-33.33 & - & - & -0.001 & 79.51 & 0.54 & 0.68 & (ألفمبة طن) \\
\hline 133.33 & - & - & 0.004 & 161.88 & 14.12 & 8.73 & (ألفترجنيه/طن) \\
\hline 100 & 0.0023 & -0.00075 & 0.003 & 129.89 & 7.37 & 5.68 & (مليون جنبه) \\
\hline
\end{tabular}

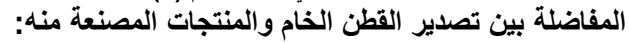

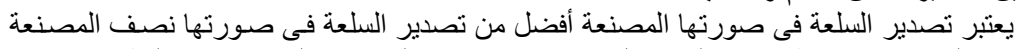

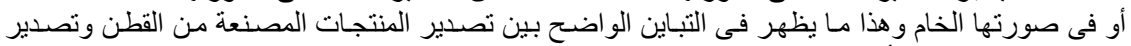

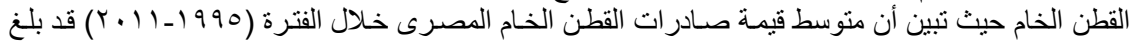

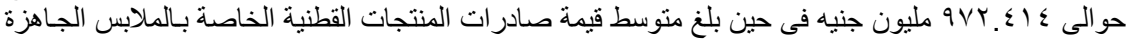

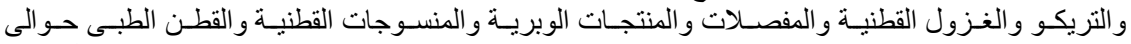

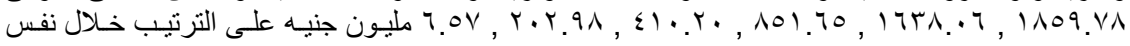

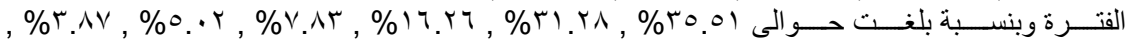

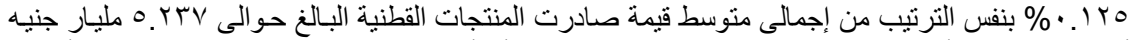

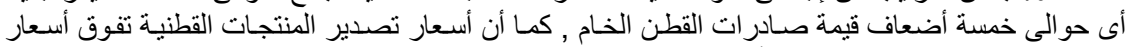

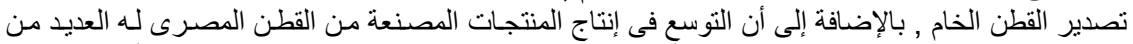

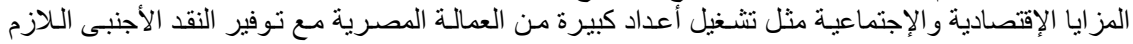

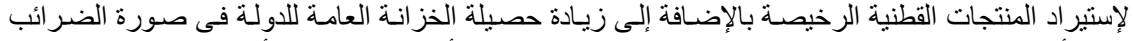

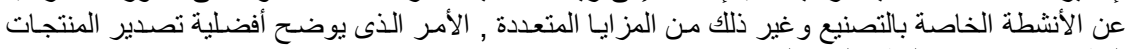

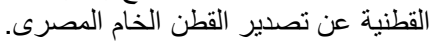

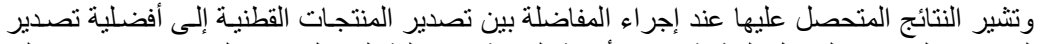

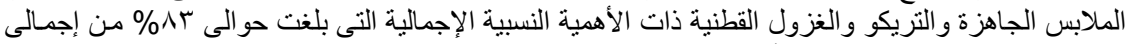

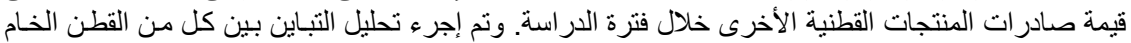

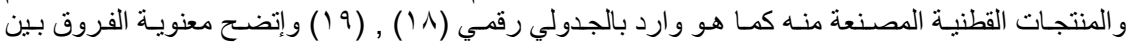
متوسطات كل منها.

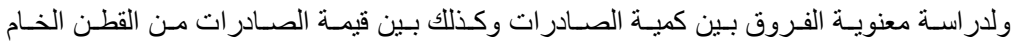

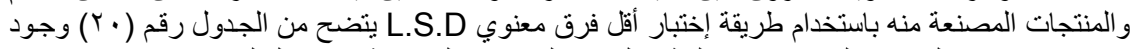

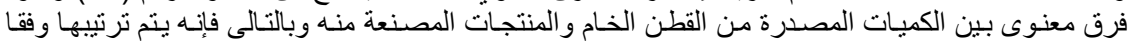
للأهمية النسبية لمتوسط الكميات المصدرة.

جدول رقم (1 ا ): تحليل التباين بين متوسط كمية الصـادرات المصرية من القطن والمنتجات المصنعة منـه

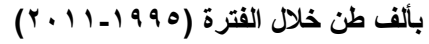


J. Agric. Econom. and Social Sci., Mansoura Univ., Vol.5 (10), October, 2014

\begin{tabular}{|l|c|c|c|c|c|}
\hline Sources of Variance & Sum of Squares & Df & Mean Square & F & Sig. \\
\hline Between Groups & 90267.767 & 7 & 12895.395 & 42.395 & .000 \\
Within Groups & 38934.380 & 128 & 304.175 & & \\
\hline Total & 129202.147 & 135 & & & \\
\hline
\end{tabular}

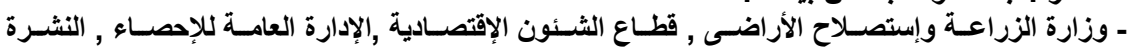
الإقتصادية، أعداد متفرقة.

ـ صندوق دعم صناعة الغزل و النسيج , الإدارة العامة للبحوث , النشرة الربع سنوية , أعداد متفرقة.

جدول رقم (9 1): نتائج تحليل التباين بين متوسط قيم الصـادرات المصرية من القطن والمنتجات المصنعة

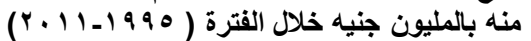

\begin{tabular}{|l|c|c|c|c|c|}
\hline Sources of Variance & Sum of Squares & Df & Mean Square & F & Sig. \\
\hline Between Groups & 55780890.874 & 7 & 7968698.696 & 17.032 & .000 \\
Within Groups & 59887253.470 & 128 & 467869.168 & & \\
\hline Total & 115668144.343 & 135 & & & \\
\hline
\end{tabular}

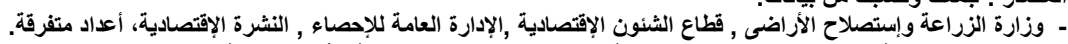

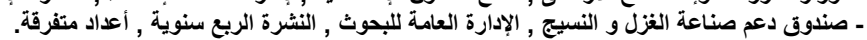

جـول رقم ( • r) : نتـائج إختبـار معنويـة الفـروق بـين متوسـط كميـات الصـادرات المصـرية مسن القطن

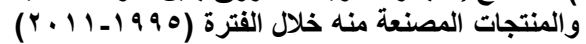

(الكمية بالألف طن)

\begin{tabular}{|c|c|c|c|c|c|c|c|c|}
\hline الطبّن & الوبنتجية & المفصنلات & الملابس الجاهزة & التريكو & المنسوجةتات & القطنية & الخامن & البيان \\
\hline & & & & & & - & 48.18 & الغزول القطنية \\
\hline & & & & & - & $29.49^{*}$ & $77.67^{*}$ & القطنية لـوجات \\
\hline & & & & - & $-19.18^{*}$ & 10.31 & $58.49^{*}$ & التركو \\
\hline & & & - & -3.09 & $-22.27^{*}$ & 7.22 & $55.40^{*}$ & الحاهلابس \\
\hline & & - & $18.29^{x}$ & $15.20^{*}$ & -3.98 & $25.51^{*}$ & $73.69^{*}$ & المفصلات \\
\hline & - & 5.57 & $23.86^{*}$ & $20.77^{\star}$ & 1.58 & $31.08^{*}$ & $79.26^{*}$ & الوبرية \\
\hline & -6.76 & $12.33^{*}$ & $30.62^{*}$ & $27.53^{*}$ & 8.35 & $37.83^{*}$ & $86.01^{*}$ & الطبي \\
\hline
\end{tabular}


Elshahawy, M. I. M. et al.

جدول رقم ( (Y) : نتائج إختبار معنوية الفروق بين متوسط قيم الصـادرات المصرية مـن القطن و المنتجـات

(القيمة بالمليون جنيه)

المصنعة منه خلال القترة (

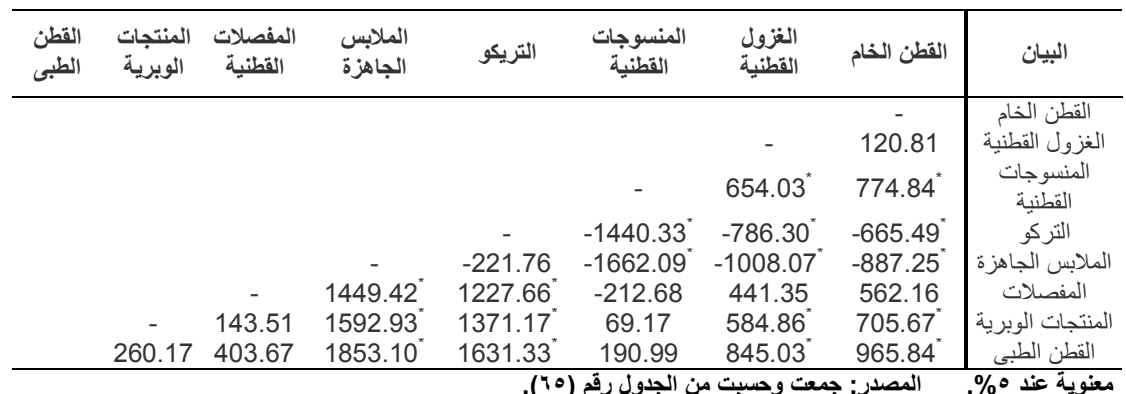

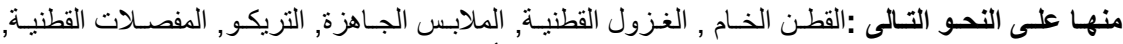

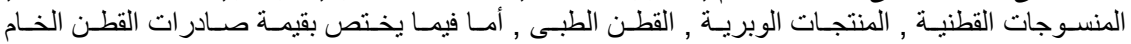

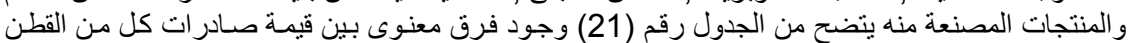

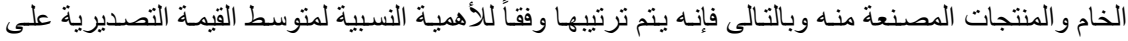

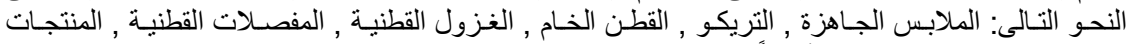

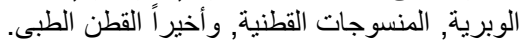

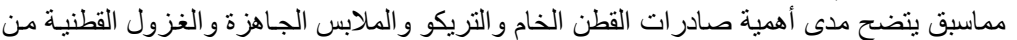

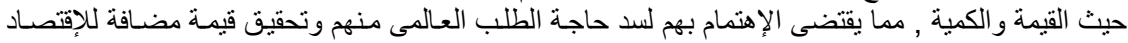

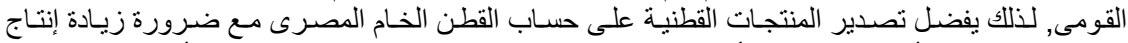

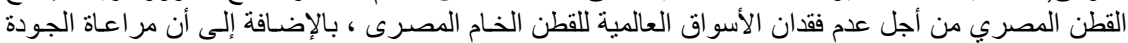

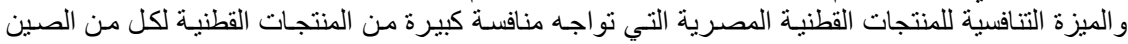

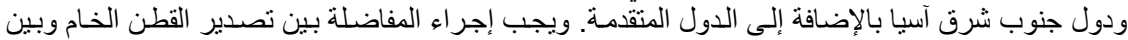

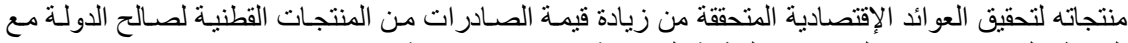

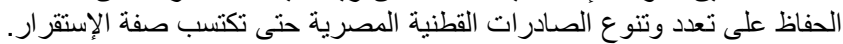

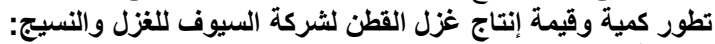

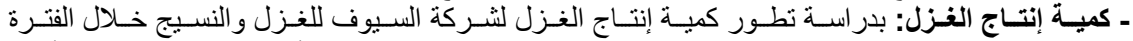

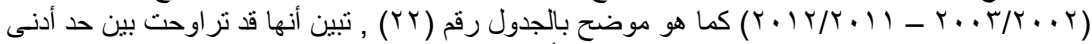

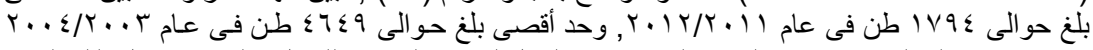

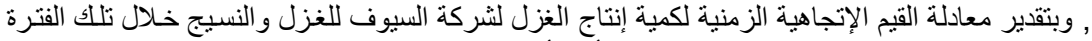

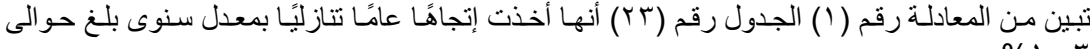
$\% 1 \cdot r$

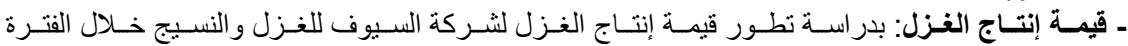

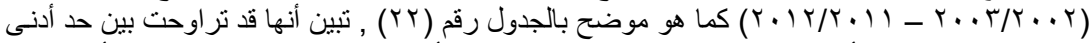

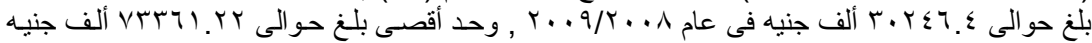

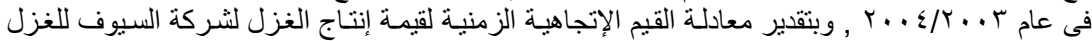

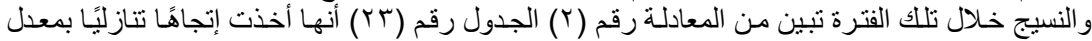

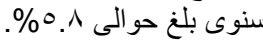

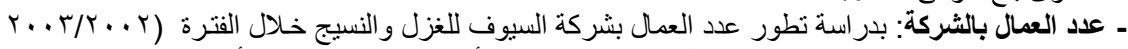
-

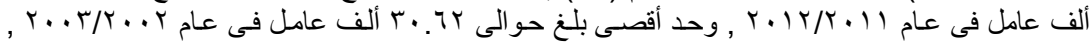

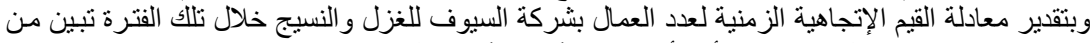

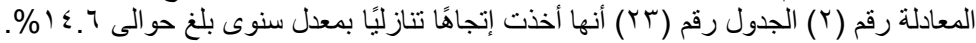




\begin{tabular}{|c|c|c|c|c|c|c|c|}
\hline \multicolumn{8}{|c|}{ 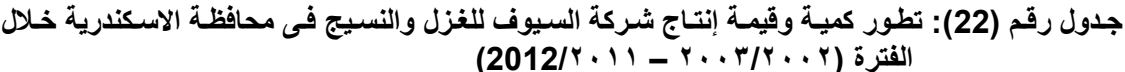 } \\
\hline 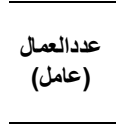 & قيمة الأجور (بالألف جنيه) & (الاف جنيمة إنتاج & إلنتاجة & قيمة الأمستخدان & (بالطن) & لية المستخدم من الأقطان & السنة \\
\hline 30618 & 18665 & 47933.96 & 4276 & 22000 & & 4030 & $2002-2003$ \\
\hline 28994 & 19349 & 73361.22 & 4649 & 43300 & & 4490 & 2003-2004 \\
\hline 28350 & 19735 & 50427.04 & 3266 & 38450 & & 4298 & 2004-2005 \\
\hline 25893 & 22987 & 51957.76 & 3776 & 33670 & & 4106 & $2005-2006$ \\
\hline 24990 & 21060 & 48155.6 & 3676 & 36940 & & 4388 & $2006-2007$ \\
\hline 18956 & 22133 & 32404.32 & 2308 & 26000 & & 2801 & $2007-2008$ \\
\hline 18067 & 23533 & 30246.4 & 2176 & 24000 & & 2657 & 2008-2009 \\
\hline 10535 & 21377 & 36014.22 & 2538 & 29000 & & 3056 & $2009-2010$ \\
\hline 9947 & 17153 & 45345.6 & 2010 & 27000 & & 1818 & 2010-2011 \\
\hline 9751 & 21848 & 36489.96 & 1794 & 17700 & & 1015 & 2011-2012 \\
\hline 20610.1 & 20784 & 45233.608 & 3046.9 & 29806 & & 3266 & المتوسط \\
\hline \multicolumn{8}{|c|}{ 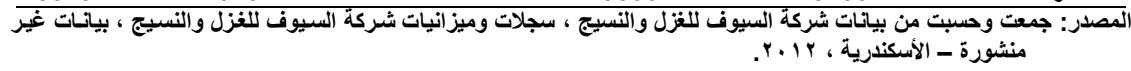 } \\
\hline \multicolumn{8}{|c|}{ 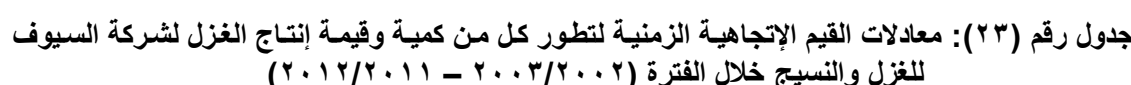 } \\
\hline No. & & quation & & & $\mathbf{R}^{2}$ & $F \quad F$ & P-Value \\
\hline 1 & $\begin{array}{l}\left.\operatorname{Ln} \hat{y}_{1}=01\right) \\
\quad(10.751\end{array}$ & $\begin{array}{l}\text { l } \leq . r Y-\cdot \\
1) \quad(-6.900\end{array}$ & $0)$ & & 0.86 & 47.61 & 0.000 \\
\hline r & $\begin{array}{r}\operatorname{Ln} y_{2}=601 \\
(6.920)\end{array}$ & $\begin{array}{r}150.880-0 \\
(-2.470\end{array}$ & 9) $058 \times$ & & - $\varepsilon r$ & $7.1 \varepsilon$ & 0.038 \\
\hline r & $\begin{array}{r}\operatorname{Ln} \hat{y}_{3}=421 \\
(6.448)\end{array}$ & $\begin{array}{r}106.738-0 \\
(-8.560\end{array}$ & $0.146 \times$ & & .9 . & VT.TV & 0.000 \\
\hline
\end{tabular}

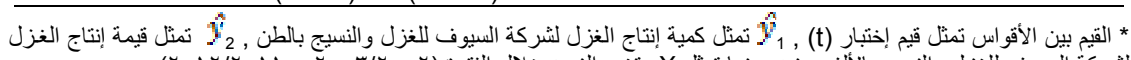

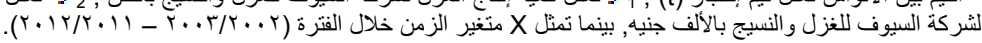
المصدر: حسبت من بيانات الجدول رقم (7) ل).

التقدير الإحصائى للالات الإنتاجية القيمية لنشاط الغزل بشركة السيوف للغزل والنسيج:

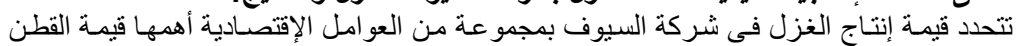

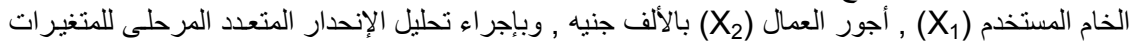

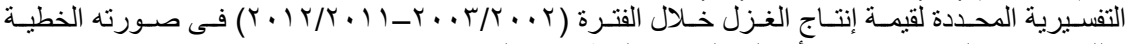
و اللو غاريتمية المزدوجة , تبين أفضلية النموذج الخطى كما يلي: $y=9730.545+1.191 X_{1} \quad \mathrm{R}^{2}=0.58 \quad \mathrm{~F}=11.15 \quad$ P-Value $=0.010$ (3.340)

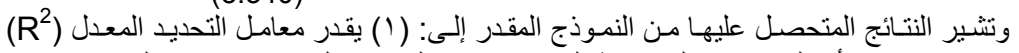

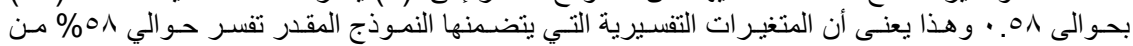

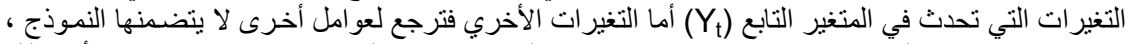

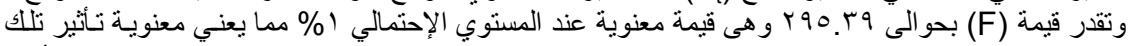

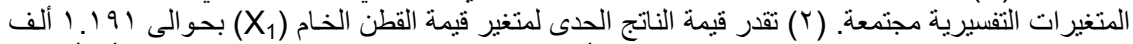

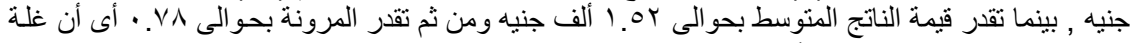

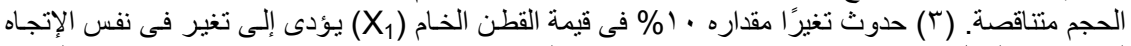

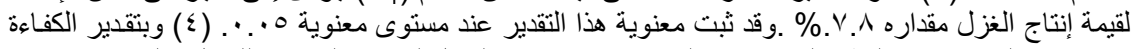

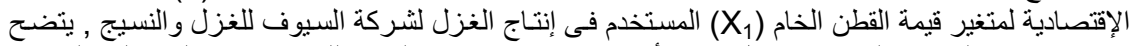

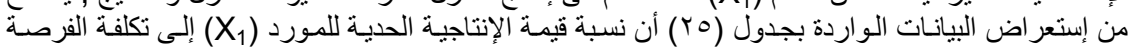


Elshahawy, M. I. M. et al.

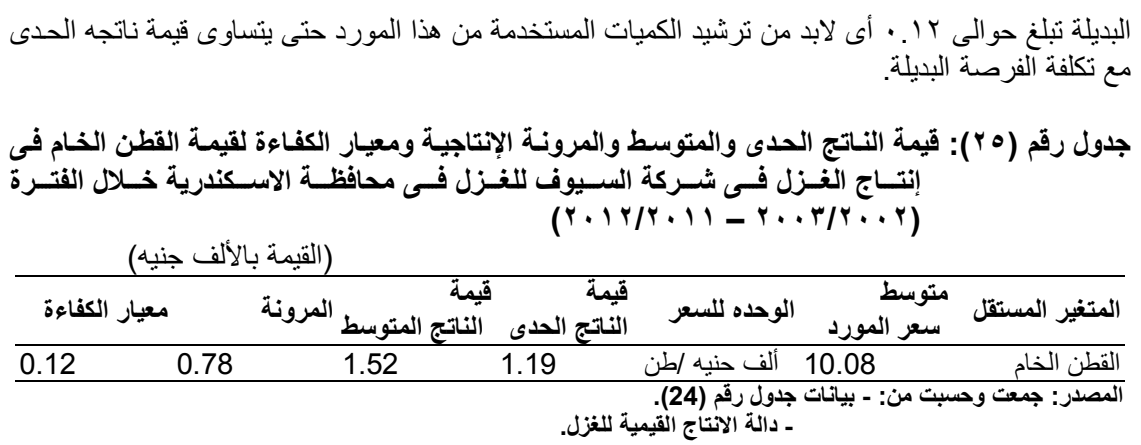

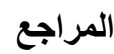

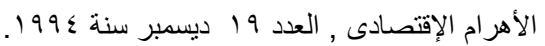

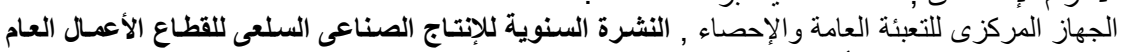
والقطاع الخاص , أعداد متفرقة . لإنة .

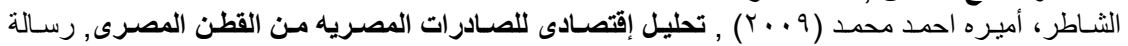

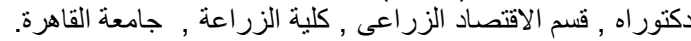

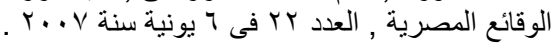

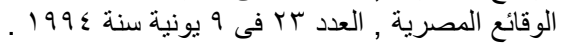

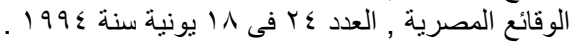

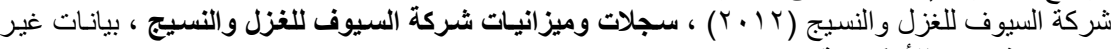
منشورة ، الأسكندرية.

صندوق دعم صناعة الغزل و النسيج , الإدارة العامة للبحوث , النشرة ربعة ربع سنوية , أعداد متفرقة.

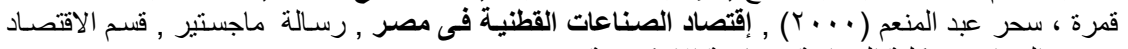

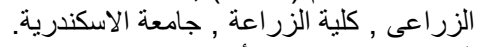

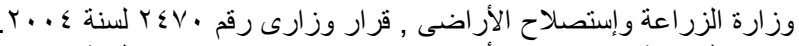

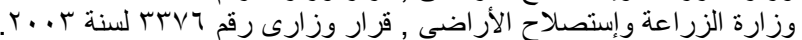

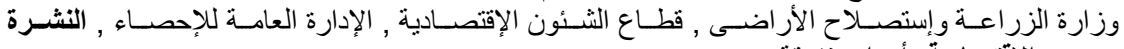
الإقتصادية، أعداد متفرقة.

Wooldridge, J., (2008). Introductory Econometrics: A Modern Approach.4th edition, South-Western College Publishers. 


\title{
ECONOMIC ANALYSIS FOR THE MOST IMPORTANT PRODUCTS MANUFACTURED FROM EGYPTIAN COTTON: A CASE STUDY: SUOF COMPANY FOR SPINNING AND WEAVING IN ALEXANDRIA \\ Elshahawy, M. I. M.;Dina M. El-Shaaer,and I. M. Ali \\ Department of Agricultural Economics-Faculty of Agriculture (Saba Basha) - Alexandria University
}

\begin{abstract}
The research aims to study the economics of products manufactured from Egyptian cotton and studying of the extent of the comparison between the export of raw cotton and manufactured products thereof in addition to some measure of efficiency indicators swords Company for Spinning and Weaving Alexandria. To achieve this research relied on some standard analysis tools, using some of the methods of statistical and mathematical models.

The results of the study showed the following: (1) The average value of the industrial production of commodity products for the textile and clothing to the private sector, about five times the value of the production of the public sector and this means the superiority of the private sector to the public sector and the decline of the role of the textile companies and apparel for the public sector in advancing the development and growth the country's economic. ((2) Decreasing the amount of exports of cotton yarn at an annual rate of about $3.2 \%$, but the value of exports increased annual growth rate of about $3.3 \%$ was due to the increase in the export price of yarn by about $6.5 \%$ per annum. (3) Event fluctuation and instability in the value of textile exports has contributed to decreasing in the quantity of exports of cotton textiles, and this can be seen lower external demand on cotton textiles, which confirms the weakness of their competitiveness in the global market. (4) It turns out the importance of the garment industry cotton in support of the Egyptian trade balance due to the rise in the value of their exports reaching about 5.0489 billion pounds in 2011, also notes that the garments sector was not affected by an increase the price of raw cotton, because it depends on the yarn imported, and the special factors the quality and prices of raw cotton in front of dwindling influence factors on levels of tastes and designs and technology used in manufacturing. (5) show that the amount of product exports hinges cotton took an upward trend during the study period, an annual growth rate of about $8.70 \%$, and the price of export products hinges cotton taking an upward trend annual growth rate of about $6.20 \%$, resulting in increased value of exports of products hinges cotton at a rate of annual growth of about $14.90 \%$. (6) that the amount of product exports fluffy cotton took an upward trend during the study period, an annual growth rate of about $8.30 \%$, and the price of export products hairy taking an upward trend annual growth rate of about $6.20 \%$, resulting in increased value of exports of products hairy annual growth rate about $14.50 \%$. (7) that the amount of cotton exports Medical passed through several stages during the study period between increases and decreases where there is as much as the amount of change during the study period by about -0.123 million melodic, while taking the export price of cotton Medical upward trend annual growth rate of about $4.5 \%$, which led that the value of cotton exports passed through several stages of Medical during the study period between increases and decreases the point where there is a coup, and as much as the amount of change during the study period by about -0.82 million melodic. (8) it became clear how important exports of raw cotton and knitted garments and cotton yarn in terms of value and quantity, and prefer to export cotton products at the expense of Egyptian raw
\end{abstract}


Elshahawy, M. I. M. et al.

cotton with the need to increase the production of Egyptian cotton in order not to lose the global markets for raw cotton of Egypt, in addition to taking into account the quality and feature competitiveness of Egyptian cotton products facing significant competition from cotton products for both China and Southeast Asian countries as well as developed countries. (9) estimating the economic efficiency of the variable value of raw cotton used in the production of yarn for the company swords for Spinning and Weaving, shows that the proportion of the value of marginal productivity to the opportunity cost of about 0.12 ie must rationalize the quantities used this resource even equal to the value of its output marginal with the opportunity cost.

The study recommends the need to encourage the private sector in this field with special attention to factors tastes and levels of design and technology used in the manufacturing side of the quality and prices of cotton, and work to increase the competitive advantage of cotton products manufactured with the need to rationalize the use of raw cotton used and optimal use.

Keywords: cotton products, economic efficiency, the combined effect, legislation and international conventions 